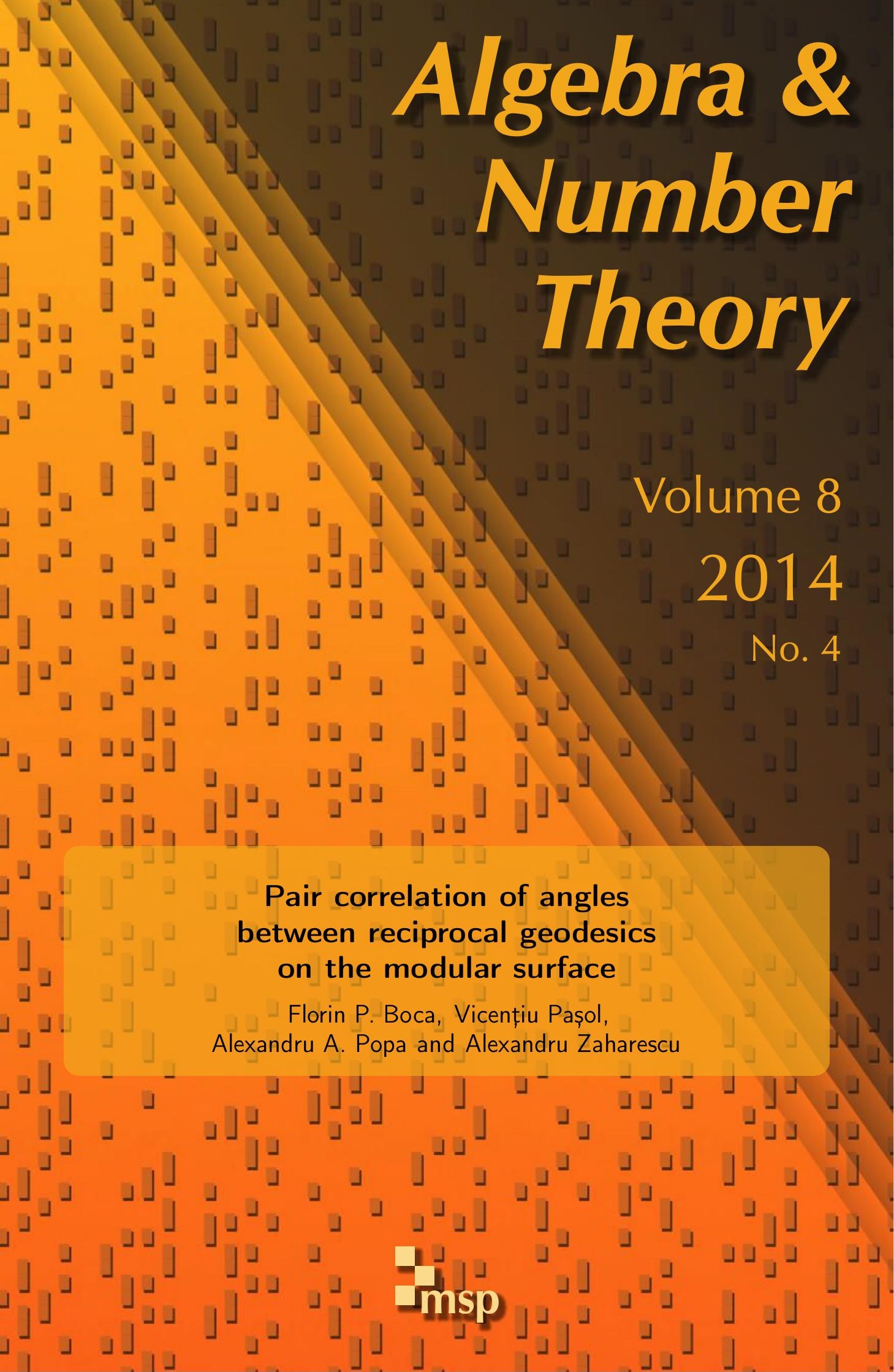




\title{
Pair correlation of angles between reciprocal geodesics on the modular surface
}

\author{
Florin P. Boca, Vicențiu Pașol, \\ Alexandru A. Popa and Alexandru Zaharescu
}

\begin{abstract}
The existence of the limiting pair correlation for angles between reciprocal geodesics on the modular surface is established. An explicit formula is provided, which captures geometric information about the length of reciprocal geodesics, as well as arithmetic information about the associated reciprocal classes of binary quadratic forms. One striking feature is the absence of a gap beyond zero in the limiting distribution, contrasting with the analog Euclidean situation.
\end{abstract}

\section{Introduction}

Let $\mathbb{W}$ denote the upper half-plane and $\Gamma=\mathrm{PSL}_{2}(\mathbb{Z})$ the modular group. Consider the modular surface $X=\Gamma \backslash \mathbb{H}$, and let $\Pi: \mathbb{W} \rightarrow X$ be the natural projection. The angles on the upper half-plane $\mathbb{H}$ considered in this paper are the same as the angles on $X$ between the closed geodesics passing through $\Pi(i)$ and the image of the imaginary axis. These geodesics were first introduced in connection with the associated "self-inverse classes" of binary quadratic forms in the classical work of Fricke and Klein [1892, p. 164], and the primitive geodesics among them were studied recently and called reciprocal geodesics by Sarnak [2007]. The aim of this paper is to establish the existence of the pair correlation measure of their angles and to explicitly express it.

For $g \in \Gamma$, denote by $\theta_{g} \in[-\pi, \pi]$ the angle between the vertical geodesic $[i, 0]$ and the geodesic ray $[i, g i]$. For $z_{1}, z_{2} \in \mathbb{U}$, let $d\left(z_{1}, z_{2}\right)$ denote the hyperbolic distance, and set

$$
\|g\|^{2}=2 \cosh d(i, g i)=a^{2}+b^{2}+c^{2}+d^{2} \quad \text { for } g=\left(\begin{array}{ll}
a & b \\
c & d
\end{array}\right) \in \mathrm{SL}_{2}(\mathbb{R}) .
$$

MSC2010: primary 11K36; secondary 11J71, 11M36, 11P21, 37D40.

Keywords: modular surface, reciprocal geodesics, pair correlation, hyperbolic lattice points,

hyperbolic lattice angles. 
It was proved by Nicholls [1983] (see also [Nicholls 1989, Theorem 10.7.6]) that for any discrete subgroup $\Gamma$ of finite covolume in $\operatorname{PSL}_{2}(\mathbb{R})$, the angles $\theta_{\gamma}$ are uniformly distributed, in the sense that for any fixed interval $I \subseteq[-\pi, \pi]$,

$$
\lim _{R \rightarrow \infty} \frac{\#\left\{\gamma \in \Gamma: \theta_{\gamma} \in I, d(i, \gamma i) \leqslant R\right\}}{\#\{\gamma \in \Gamma: d(i, \gamma i) \leqslant R\}}=\frac{|I|}{2 \pi} .
$$

Effective estimates for the rate of convergence that allow one to take $|I| \asymp e^{-c R}$ as $R \rightarrow \infty$ for some constant $c=c_{\Gamma}>0$ were proved for $\Gamma=\Gamma(N)$ by one of us [Boca 2007], and in general situations by Risager and Truelsen [2010] and by Gorodnik and Nevo [2012]. Other related results concerning the uniform distribution of real parts of orbits in hyperbolic spaces were proved by Good [1983], and more recently by Risager and Rudnick [2009].

The statistics of spacings, such as the pair correlation or the nearest neighbor distribution (also known as the gap distribution) measure the fine structure of sequences of real numbers in a more subtle way than the classical Weyl uniform distribution. Very little is known about the spacing statistics of closed geodesics. In fact, the only result that we are aware of, due to Pollicott and Sharp [2006], concerns the correlation of differences of lengths of pairs of closed geodesics on a compact surface of negative curvature, ordered with respect to the word length on the fundamental group.

This paper investigates the pair correlation of angles $\theta_{\gamma}$ with $d(i, \gamma i) \leqslant R$, or equivalently with $\|\gamma\|^{2} \leqslant Q^{2}=e^{R} \sim 2 \cosh R$ as $Q \rightarrow \infty$. As explained in Section 2, these are exactly the angles between reciprocal geodesics on the modular surface.

The Euclidean analog of this problem considers the angles between the line segments connecting the origin $(0,0)$ with all integer points $(m, n)$ satisfying $m^{2}+n^{2} \leqslant Q^{2}$ as $Q \rightarrow \infty$. When only primitive lattice points are being considered (rays are counted with multiplicity one), the problem reduces to the study of the pair correlation of the sequence of Farey fractions with the $L^{2}$ norm $\|m / n\|_{2}^{2}=m^{2}+n^{2}$. Its pair correlation function is plotted on the left of Figure 1. When Farey fractions are ordered by their denominator, the pair correlation is shown to exist and it is explicitly computed in [Boca and Zaharescu 2005]. A common important feature is the existence of a gap beyond zero for the pair correlation function. This is an ultimate reflection of the fact that the area of a nondegenerate triangle with integer vertices is at least $\frac{1}{2}$, which corresponds to the familiar inequality $|b / d-a / c| \geqslant 1 / c d$ satisfied by two lattice points $P=(a, b)$ and $Q=(c, d)$ with $\operatorname{Area}(\triangle O P Q)>0$.

For the hyperbolic lattice centered at $i$, it is convenient to start with the (nonuniformly distributed) numbers $\tan \left(\theta_{\gamma} / 2\right)$ with multiplicities, rather than the angles $\theta_{\gamma}$ themselves. Employing obvious symmetries explained in Section 3, it is further convenient to restrict to a set of representatives $\Gamma_{I}$ consisting of matrices $\gamma$ with nonnegative entries such that the point $\gamma i$ is in the first quadrant in Figure 2. The 
pair correlation measures of the finite set $\mathfrak{A}_{Q}$ of elements $\theta_{\gamma}$ with $\gamma \in \Gamma_{\mathbf{I}}$ and $\|\gamma\| \leqslant Q$ (counted with multiplicities) is defined as

$$
R_{Q}^{\mathfrak{A}}(\xi)=\frac{1}{B_{Q}} \#\left\{\left(\gamma, \gamma^{\prime}\right) \in \Gamma_{\mathbf{I}}^{2}:\|\gamma\|,\left\|\gamma^{\prime}\right\| \leqslant Q, \gamma^{\prime} \neq \gamma, 0 \leqslant \frac{2}{\pi}\left(\theta_{\gamma^{\prime}}-\theta_{\gamma}\right) \leqslant \frac{\xi}{B_{Q}}\right\},
$$

where $B_{Q} \sim \frac{3}{8} Q^{2}$ denotes the number of elements $\gamma \in \Gamma_{\mathbf{I}}$ with $\|\gamma\| \leqslant Q$. As it will be used in the proof, we similarly define the pair correlation measure $R_{Q}^{\mathfrak{T}}(\xi)$ of the set $\mathfrak{T}_{Q}$ of elements $\tan \left(\theta_{\gamma} / 2\right)$ with $\gamma \in \Gamma_{\mathbf{I}}$ and $\|\gamma\| \leqslant Q$.

One striking feature, illustrated by the numerical calculations in Figure 1, points to the absence of a gap beyond zero in the limiting distribution, in contrast with the analog Euclidean situation.

The main result of this paper is the proof of existence and explicit computation of the pair correlation measure $R_{2}^{\mathfrak{A}}$ given by

$$
R_{2}^{\mathfrak{A}}(\xi)=R_{2}^{\mathfrak{A}}((0, \xi]):=\lim _{Q \rightarrow \infty} R_{Q}^{\mathfrak{A}}(\xi),
$$

and similarly for $R_{2}^{\mathfrak{T}}$, thus answering a question raised in [Boca 2007].

To give a precise statement, consider $\mathfrak{S}$, the free semigroup on two generators $L=\left(\begin{array}{ll}1 & 0 \\ 1 & 1\end{array}\right)$ and $R=\left(\begin{array}{ll}1 & 1 \\ 0 & 1\end{array}\right)$. Repeated application of the Euclidean algorithm shows that $\mathfrak{S} \cup\{I\}$ coincides with the set of matrices in $\mathrm{SL}_{2}(\mathbb{Z})$ with nonnegative entries. The explicit formula for $R_{2}^{\mathfrak{T}}(\xi)$ is given as a series of volumes summed over $\mathfrak{S}$, plus a finite sum of volumes, and it is stated in Theorem 2 (Section 7). The formula for $R_{2}^{\mathfrak{T}}(\xi)$ leads to an explicit formula for $R_{2}^{\mathfrak{A}}(\xi)$, which we state here, partly because the pair correlation function for the angles $\theta_{\gamma}$ is more interesting, being equidistributed, and partly because the formula we obtain is simpler.

Theorem 1. The pair correlation measure $R_{2}^{\mathfrak{A}}$ on $[0, \infty)$ exists and is given by the $C^{1}$ function

$$
R_{2}^{\mathfrak{A}}\left(\frac{3}{4 \pi} \xi\right)=\frac{8}{3 \zeta(2)}\left(\sum_{M \in \mathfrak{S}} B_{M}(\xi)+\sum_{\ell \in[0, \xi / 2)} \sum_{K \in[1, \xi / 2)} A_{K, \ell}(\xi)\right) .
$$

For $M \in \mathfrak{S}$, letting $U_{M}=\|M\|^{2} / \sqrt{\|M\|^{4}-4}, \theta_{M}$ as above, and $f_{+}=\max \{f, 0\}$, we have

$$
B_{M}(\xi)=\frac{\pi}{4} \int_{0}^{\pi / 2} \frac{\left(1 / \sqrt{\|M\|^{4}-4}-\sin \left(2 \theta-\theta_{M}\right) / \xi\right)_{+}}{U_{M}+\cos \left(2 \theta-\theta_{M}\right)} d \theta .
$$

For integers $\ell \in[0, \xi / 2), K \in[1, \xi / 2)$, we have

$$
A_{K, \ell}(\xi)=\int_{0}^{\pi / 4} A_{K, \ell}\left(\frac{\xi}{2 \cos ^{2} t}, t\right) \frac{d t}{\cos ^{2} t},
$$


where $A_{K, \ell}(\xi, t)$ is the area of the region defined by those $r e^{i \theta} \in[0,1]^{2}$ such that

$$
L_{\ell+1}\left(e^{i \theta}\right)>0, \quad \frac{F_{K, \ell}(\theta)}{\xi} \leqslant r^{2} \leqslant \frac{\cos ^{2} t}{\max \left\{1, L_{\ell}^{2}\left(e^{i \theta}\right)+L_{\ell+1}^{2}\left(e^{i \theta}\right)\right\}},
$$

with $e^{i \theta}=(\cos \theta, \sin \theta)$, the piecewise linear functions $L_{i}$ as defined in (5-5), and

$$
F_{K, \ell}(\theta):=\cot \theta+\sum_{i=1}^{\ell} \frac{1}{L_{i-1}\left(e^{i \theta}\right) L_{i}\left(e^{i \theta}\right)}+\frac{L_{\ell+1}\left(e^{i \theta}\right)}{L_{\ell}\left(e^{i \theta}\right)\left(L_{\ell}^{2}\left(e^{i \theta}\right)+L_{\ell+1}^{2}\left(e^{i \theta}\right)\right)} .
$$

Rates of convergence in (1-1) are effectively described in the proof of Theorem 2 and in Proposition 15.

When $\xi \leqslant 2$, the second sum in (1-2) disappears and the derivative $B_{M}^{\prime}(\xi)$ is explicitly computed in Lemma 17 , yielding an explicit formula for the pair correlation density function $g_{2}^{\mathfrak{A}}(\xi)=d R_{2}^{\mathfrak{A}}(\xi) / d \xi$, which matches the graph in Figure 1.

Corollary 1. For $0<\xi \leqslant 2$ we have

$$
g_{2}^{\mathfrak{A}}\left(\frac{3}{4 \pi} \xi\right)=\frac{16}{3 \xi^{2}} \sum_{M \in \mathfrak{S}} \ln \left(\frac{\|M\|^{2}+\sqrt{\|M\|^{4}-4}}{\|M\|^{2}+\sqrt{\|M\|^{4}-4-\xi^{2}}}\right) .
$$

A formula valid for $0<\xi \leqslant 4$ is given in (8-11) after computing $A_{0, K}^{\prime}(\xi)$.
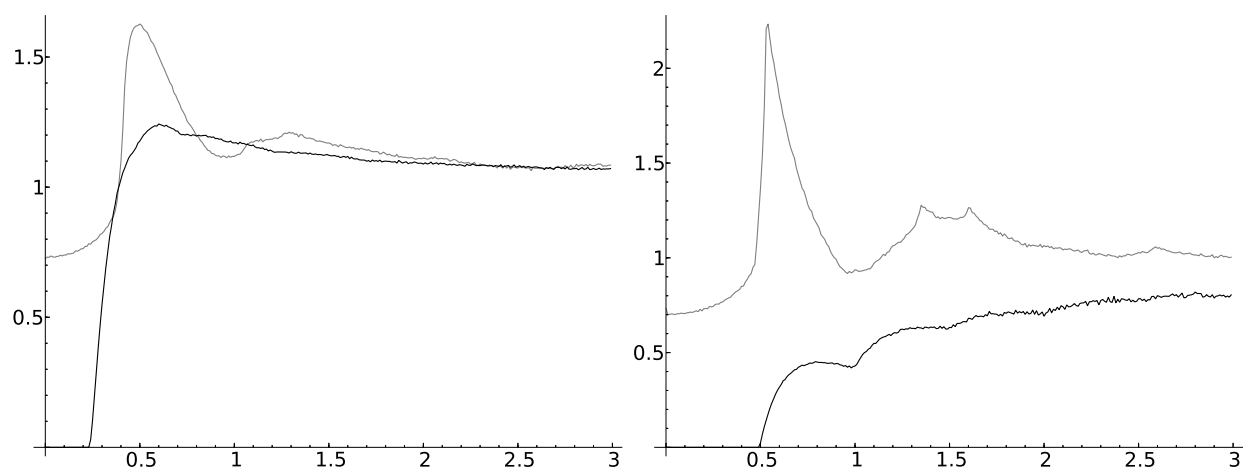

Figure 1. The pair correlation functions $g_{2}^{\mathfrak{T}}$ (left) and $g_{2}^{\mathfrak{A}}$ (right), plotted in gray, compared with the pair correlation function of Farey fractions with $L^{2}$ norm (left), and of the angles (with multiplicities) of lattice points in Euclidean balls (right). The graphs are obtained by counting the pairs in their definition, using $Q=4000$, for which $B_{Q}=6000203$. We used Magma [Bosma et al. 1997] for the numerical computations, and SAGE [Stein et al. 2012] for plotting the graphs. 
The computation is performed in Section 8.2, and it identifies the first spike in the graph of $g_{2}^{\mathfrak{A}}(x)$ at $x=(3 / 4 \pi) \sqrt{5}$. A proof of an explicit formula for the pair correlation density $g_{2}^{\mathfrak{A}}(x)$ valid for all $x$, and working also when the point $i$ is replaced by the other elliptic point $\rho=e^{\pi i / 3}$, will be given in [Boca et al. 2013].

Since the series in Corollary 1 is dominated by the absolutely convergent sum $\sum_{M} \xi^{2}\|M\|^{-4}$, we can take the limit as $\xi \rightarrow 0$ :

$$
g_{2}^{\mathfrak{A}}(0)=\frac{2}{3} \sum_{M \in \mathfrak{S}}\left(\frac{\|M\|^{2}}{\sqrt{\|M\|^{4}-4}}-1\right)=0.7015 \ldots
$$

Remarkably, the previous two formulas, as well as (1-2) for $\xi \leqslant 2$, can be written geometrically as a sum over the primitive closed geodesics $\mathscr{C}$ on $X$ which pass through the point $\Pi(i)$, where the summand depends only on the length $\ell(\mathscr{C})$ :

$$
g_{2}^{\mathfrak{A}}(0)=\frac{8}{3} \sum_{\mathscr{C}} \sum_{n \geqslant 1} \frac{1}{e^{n \ell(\mathscr{C})}-1} .
$$

This is proved in Section 2, where we also give an arithmetic version based on an explicit description of the reciprocal geodesics $\mathscr{b}$ due to Sarnak [2007].

For the rest of the introduction we sketch the main ideas behind the proof, describing also the organization of the article. After reducing to angles in the first quadrant in Section 3, we show that the pair correlation of the quantities $\Psi(\gamma)=\tan \left(\theta_{\gamma} / 2\right)$ is identical to that of $\Phi(\gamma)=\operatorname{Re}(\gamma i)$. We are led to estimating the cardinality of the set

$$
\left\{\left(\gamma, \gamma^{\prime}\right) \in \Gamma_{\mathbf{I}}^{2}:\|\gamma\|,\left\|\gamma^{\prime}\right\| \leqslant Q, \gamma^{\prime} \neq \gamma, 0 \leqslant Q^{2}\left(\Phi\left(\gamma^{\prime}\right)-\Phi(\gamma)\right) \leqslant \xi\right\} .
$$

For a matrix $\gamma=\left(\begin{array}{cc}p^{\prime} & p \\ q^{\prime} & q\end{array}\right)$ with nonnegative entries, $\|\gamma\| \leqslant Q$, and $q, q^{\prime}>0$, consider the associated Farey interval $\left[p / q, p^{\prime} / q^{\prime}\right]$, which contains $\Phi(\gamma)$. In Section 4 , we break the set of pairs $\left(\gamma, \gamma^{\prime}\right)$ above in two parts, depending on whether one of the associated Farey intervals contains the other, or the two intervals intersect at most at one endpoint. In the first case we have $\gamma=\gamma^{\prime} M$ or $\gamma^{\prime}=\gamma M$ with $M \in \mathfrak{S}$, while in the second we have a similar relation depending on the number $\ell$ of consecutive Farey fractions there are between the two intervals. The first case contributes to the series over $\mathfrak{S}$ in (1-2), while the second case contributes to the sum over $K, \ell$. The triangle map $T$ whose iterates define the piecewise linear functions $L_{i}(x, y)$, first introduced in [Boca et al. 2001], makes its appearance in the second case, being related to the denominator of the successor function for Farey fractions.

To estimate the number of pairs $(\gamma, \gamma M)$ in the first case, a key observation is that for each $M \in \Gamma$ there exists an explicit elementary function $\Xi_{M}(x, y)$, given by $(5-1)$, such that

$$
\Phi(\gamma)-\Phi(\gamma M)=\Xi_{M}\left(q^{\prime}, q\right)
$$


for $\gamma$ as above. Together with estimates for the number of points in two dimensional regions based on bounds on Kloosterman sums (Lemma 7), this allows us to estimate the number of pairs $(\gamma, \gamma M)$ with fixed $M \in \mathfrak{S}$, in terms of the volume of a three dimensional body $S_{M, \xi}$ given in (7-14). The absence of a gap beyond zero in the pair correlation measure arises as a result of this estimate. The details of the calculation are given in Section 7, leading to an explicit formula for $R_{2}^{\mathfrak{T}}$ (Theorem 2).

Finally in Section 8 we pass to the pair correlation of the angles $\theta_{\gamma}$, obtaining the formulas of Theorem 1 and Corollary 1.

In this paper we focus on the full modular lattice centered at $i$, both because of the arithmetic connection with reciprocal geodesics, and because in this case the connection between unimodular matrices and Farey intervals is most transparent. It is this connection and the intuition provided by the repulsion of Farey fractions that guides our argument, and leads to the explicit formula for the pair correlation function, which is the first of this kind for hyperbolic lattices.

In a subsequent paper [Boca et al. 2013], we abstract some of this intuition and propose a different conjectural formula for the pair correlation function of an arbitrary lattice in $\mathrm{PSL}_{2}(\mathbb{R})$, centered at a point on the upper half plane, which we prove for the full level lattice centered at elliptic points. While the formula in that paper is more general, the method of proof, and the combinatorial-geometric intuition behind it, is reflected more accurately in the formula of Theorem 1: the infinite sum in the formula corresponds to pairs of matrices where there is no repulsion between their Farey intervals, while the finite sum corresponds to pairs of matrices where there is repulsion. The approach used in [Boca et al. 2013] builds on the estimates and method of the present paper.

A proof of that paper's conjecture by spectral methods has been proposed in a preprint by Kelmer and Kontorovich [2013]. By comparison, our approach is entirely elementary (using only standard bounds on Kloosterman sums), and via the repulsion argument it provides a natural way of approximating the pair correlation function. A key insight in the present paper, which is also the starting point of [Boca et al. 2013] and [Kelmer and Kontorovich 2013], is that instead of counting pairs $\left(\gamma, \gamma^{\prime}\right) \in \Gamma \times \Gamma$ in the definition of the pair correlation measure, we fix a matrix $M$, count pairs $(\gamma, \gamma M)$, and sum over $M$. The same approach may prove useful for the pair correlation problem for lattices in other groups as well.

\section{Reciprocal geodesics on the modular surface}

In this section we recall the definition of reciprocal geodesics and explain how the pair correlation of the angles they make with the imaginary axis is related to the pair correlation considered in the introduction. We also show that the sums over the semigroup $\mathfrak{S}$ appearing in the introduction can be expressed geometrically in 
terms of sums over primitive reciprocal geodesics. A description of the trajectory of reciprocal geodesics on the fundamental domain seems to have first appeared in the classical work of Fricke and Klein [1892, p.164], where it is shown that they consist of two closed loops, one the reverse of the other. There the terminology "sich selbst inverse Classe" is used for the equivalence classes of quadratic forms corresponding to reciprocal conjugacy classes of hyperbolic matrices.

Oriented closed geodesics on $X$ are in one-to-one correspondence with conjugacy classes $\{\gamma\}$ of hyperbolic elements $\gamma \in \Gamma$. To a hyperbolic element $\gamma \in \Gamma$ one attaches its axis $a_{\gamma}$ on $\mathbb{U}$, namely the semicircle whose endpoints are the fixed points of $\gamma$ on the real axis. The part of the semicircle between $z_{0}$ and $\gamma z_{0}$, for any $z_{0} \in a_{\gamma}$, projects to a closed geodesic on $X$, with multiplicity one if and only if $\gamma$ is a primitive matrix (not a power of another hyperbolic element of $\Gamma$ ). The group that fixes the semicircle $a_{\gamma}$ (or equivalently its endpoints on the real axis) is generated by one primitive element $\gamma_{0}$.

We are concerned with (oriented) closed geodesics passing through $\Pi(i)$ on $X$. Since the axis of a hyperbolic element $A$ passes through $i$ if and only if $A$ is symmetric, the closed geodesics passing through $\Pi(i)$ correspond to the set $\mathscr{R}$ of hyperbolic conjugacy classes $\{\gamma\}$ which contain a symmetric matrix. The latter are exactly the reciprocal geodesics considered in [Sarnak 2007], where only primitive geodesics are considered.

The reciprocal geodesics can be parametrized in a two-to-one manner by the set $\mathfrak{S} \subset \Gamma$, defined in the introduction, which consists of matrices distinct from the identity with nonnegative entries. To describe this correspondence, let $\mathscr{A} \subset \Gamma$ be the set of symmetric hyperbolic matrices with positive entries. Then we have maps

$$
\mathfrak{S} \rightarrow \mathscr{A} \rightarrow \mathscr{R}
$$

where the first map takes $\gamma \in \mathfrak{S}$ to $A=\gamma \gamma^{t}$, and the second takes the hyperbolic symmetric $A$ to its conjugacy class $\{A\}$. The first map is bijective, while the second is two-to-one and onto, as follows from [Sarnak 2007]. More precisely, if $A=\gamma \gamma^{t} \in \mathscr{A}$ is a primitive matrix, then $B=\gamma^{t} \gamma \neq A$ is the only other matrix in $\mathscr{A}$ conjugate to $A$, and $\left\{A^{n}\right\}=\left\{B^{n}\right\}$ for all $n \geqslant 0$.

Note also that $\|\gamma\|^{2}=\operatorname{Tr}\left(\gamma \gamma^{t}\right)$, and if $A$ is hyperbolic with $\operatorname{Tr}(A)=T$, then the length of the geodesic associated to $\{A\}$ is $2 \ln N(A)$ with $N(A)=\frac{1}{2}\left(T+\sqrt{T^{2}-4}\right)$.

Lemma 2. Let $A \in \Gamma$ be a hyperbolic symmetric matrix and let $\gamma \in \Gamma$ such that $A=\gamma \gamma^{t}$. Then the point $\gamma i$ is halfway (in hyperbolic distance) between $i$ and $A i$ on the axis of $A$.

Proof. We have $d(i, \gamma i)=d\left(i, \gamma^{t} i\right)=d(\gamma i, A i)$ where the first equality follows from the hyperbolic distance formula and the second since $\Gamma$ acts by isometries 
on $\mathbb{H}$. Using formula (3-3), one checks that the angles of $i, \gamma i$ and $i, A i$ are equal, hence $\gamma i$ is indeed on the axis of $A$.

We can now explain the connection between the angles $\theta_{\gamma}$ in the first and second quadrant in Figure 2, and the angles made by the reciprocal geodesics with the image $\Pi(i \rightarrow i \infty)=\Pi(i \rightarrow 0)$. Namely, points in the first and second quadrant are parametrized by $\gamma i$ with $\gamma \in \mathfrak{S}$, and by the lemma the reciprocal geodesic corresponding to $A=\gamma \gamma^{t} \in \mathscr{A}$ consists of the loop $\Pi(i \rightarrow \gamma i)$, followed by $\Pi\left(i \rightarrow \gamma^{t} i\right)$ (which is the same as the reverse of the first loop). Therefore to each reciprocal geodesic corresponding to $A=\gamma \gamma^{t} \in \mathscr{A}$ correspond two angles, those attached to $\gamma i$ and $\gamma^{t} i$ in Figure 2, measured in the first or second quadrant so that all angles are between 0 and $\pi / 2$.

In conclusion, the angles made by the reciprocal geodesics on $X$ with the fixed direction $\Pi(i \rightarrow i \infty)$ consist of the angles in the first quadrant considered before, each appearing twice. Ordering the points $\gamma i$ in the first quadrant by $\|\gamma\|$ corresponds to ordering the geodesics by their length. Therefore the pair correlation measure of the angles of reciprocal geodesics is $2 R_{2}^{\mathfrak{A}}(\xi / 2)$, where $R_{2}^{\mathfrak{A}}$ was defined in the introduction.

The parametrization (2-1) of reciprocal geodesics allows one to rewrite the series appearing in the formula for $g_{2}^{\mathfrak{A}}(0)$ in the introduction, as a series over the primitive reciprocal classes $\mathscr{R}^{\text {prim: }}$

$$
\sum_{M \in \mathfrak{S}}\left(\frac{\|M\|^{2}}{\sqrt{\|M\|^{4}-4}}-1\right)=\sum_{A \in \mathscr{A}} \frac{2}{N(A)^{2}-1}=4 \sum_{\{\gamma\} \in \mathscr{R} \text { prim }} \sum_{n \geqslant 1} \frac{1}{N(\gamma)^{2 n}-1},
$$

where we have used the fact that for a hyperbolic matrix $A$ of trace $T$ we have

$$
\sqrt{T^{2}-4}=N(A)-N(A)^{-1} \text { and } N\left(A^{n}\right)=N(A)^{n} .
$$

One can rewrite the sum further using the arithmetic description of primitive reciprocal geodesics given in [Sarnak 2007]. Namely, let $\mathscr{D}_{\mathscr{R}}$ be the set of nonsquare positive discriminants $2^{\alpha} D^{\prime}$ with $\alpha \in\{0,2,3\}$ and $D^{\prime}$ odd divisible only by primes $p \equiv 1(\bmod 4)$. Then the set of primitive reciprocal classes $\mathscr{R}^{\text {prim }}$ decomposes as a disjoint union of finite sets:

$$
\mathscr{R}^{\text {prim }}=\bigcup_{d \in \mathscr{D}_{\mathscr{R}}} \mathscr{R}_{d}^{\text {prim }}
$$

with $\left|\mathscr{R}_{d}^{\text {prim }}\right|=v(d)$, the number of genera of binary quadratic forms of discriminant $d$. If $d \in \mathscr{D}_{\mathscr{R}}$ has exactly $\lambda$ odd prime factors, $v(d)$ equals $2^{\lambda}$ if 8 divides $d$ and $2^{\lambda-1}$ otherwise. Each class $\{\gamma\} \in \mathscr{R}_{d}^{\text {prim }}$ has

$$
N(\gamma)=\alpha_{d}=\frac{1}{2}\left(u_{0}+v_{0} \sqrt{d}\right),
$$


with $\left(u_{0}, v_{0}\right)$ the minimal positive solution to Pell's equation $u^{2}-d v^{2}=4$. We then have

$$
\sum_{\{\gamma\} \in \mathscr{R}_{\text {prim }}} \sum_{n \geqslant 1} \frac{1}{N(\gamma)^{2 n}-1}=\sum_{d \in \mathscr{D}_{\mathscr{R}}} \sum_{n \geqslant 1} \frac{v(d)}{\alpha_{d}^{2 n}-1} .
$$

In the same way, by Lemma 13 the pair correlation measure $R_{2}^{\mathfrak{T}}(\xi)$ in Theorem 1 can be written for $\xi \leqslant 1$ as a sum over classes $\{\gamma\} \in \mathscr{R}^{\text {prim }}$, where each summand depends only on $\xi$ and $N(\gamma)$.

\section{Reduction to the first quadrant}

In this section we establish notation in use throughout the paper, and we reduce the pair correlation problem to angles in the first quadrant. A similar reduction can be found in [Chamizo 2006], in the context of visibility problems for the hyperbolic lattice centered at $i$.

For each matrix $g=\left(\begin{array}{ll}a & b \\ c & d\end{array}\right) \in \mathrm{SL}_{2}(\mathbb{R})$, define the quantities

$$
\begin{gathered}
X_{g}=a^{2}+b^{2}, \quad Y_{g}=c^{2}+d^{2}, \quad Z_{g}=a c+b d, \quad T_{g}=X_{g}+Y_{g}=\|g\|^{2}, \\
\Phi(g)=\operatorname{Re}(g i)=\frac{Z_{g}}{Y_{g}}, \quad \epsilon_{g}=\epsilon_{T_{g}}=\frac{1}{2}\left(T_{g}-\sqrt{T_{g}^{2}-4}\right) .
\end{gathered}
$$

The upper half-plane $\mathbb{U}$ is partitioned into four quadrants:

$$
\begin{aligned}
\mathbf{I} & =\{z \in \mathbb{H}: \operatorname{Re} z>0,|z|<1\}, \quad \mathbf{I I}=\{z \in \mathbb{H}: \operatorname{Re} z>0,|z|>1\}, \\
\mathbf{I I I} & =\{z \in \mathbb{H}: \operatorname{Re} z<0,|z|>1\}, \quad \mathbf{I V}=\{z \in \mathbb{H}: \operatorname{Re} z<0,|z|<1\} .
\end{aligned}
$$

Note that all the points $g i$ for $g \in \Gamma$ lie in one of the four open quadrants, with the exception of $i$ itself. This follows from the relation

$$
X_{g} Y_{g}-Z_{g}^{2}=1
$$

which will be often used.

In this section, simply take $X=X_{g}, Y=Y_{g}, Z=Z_{g}, \theta=\theta_{g}$. A direct calculation shows that the center of the circle through $i$ and $g i$ is $\alpha=(X-Y) /(2 Z)$, leading to

$$
\tan \theta_{g}=-\frac{1}{\alpha}=\frac{2 Z_{g}}{Y_{g}-X_{g}} \quad \text { for all } \theta_{g} \in[-\pi, \pi]
$$

Plugging this into

$\tan \frac{\theta}{2}=\frac{\tan \theta}{1+\sqrt{1+\tan ^{2} \theta}}$ if $|\theta|<\frac{\pi}{2}$ or $\tan \frac{\theta}{2}=-\frac{1+\sqrt{1+\tan ^{2} \theta}}{\tan \theta}$ if $\frac{\pi}{2}<|\theta|<\pi$, and employing (3-2) and the equivalences $|g i|<1 \Longleftrightarrow X<Y$ and $\operatorname{Re}(\gamma i)>0 \Longleftrightarrow$ $Z>0$, we find the useful formulas 


$$
\Psi(g):=\tan \frac{\theta_{g}}{2}=\frac{\sqrt{T_{g}^{2}-4}+X_{g}-Y_{g}}{2 Z_{g}}=\frac{X_{g}-\epsilon_{g}}{Z_{g}}=\frac{Z_{g}}{Y_{g}-\epsilon_{g}}
$$

for all $\theta_{g} \in[-\pi, \pi]$.

We set $\gamma=\left(\begin{array}{ll}a & b \\ c & d\end{array}\right), \tilde{\gamma}=\left(\begin{array}{ll}d & c \\ b & a\end{array}\right), s=\left(\begin{array}{cc}0 & -1 \\ 1 & 0\end{array}\right)$. Let $\gamma \in \Gamma, \gamma \neq I, s$. For $\gamma i$ to be in the right half-plane we need $\operatorname{Re}(\gamma i)>0$. This is equivalent to $a c+b d>0$, and implies that $a c \geqslant 0$ and $b d \geqslant 0$ because $a b c d=b c+(b c)^{2} \geqslant 0$. Since $a c \geqslant 0$, without loss of generality we will assume that $a \geqslant 0$ and $c \geqslant 0$ (otherwise consider $-\gamma$ instead). Without loss of generality, we will assume that $b \geqslant 0, d \geqslant 0$ as well (otherwise, we consider $-\gamma s=\left(\begin{array}{ll}-b & a \\ -d & c\end{array}\right)$ instead, since $\gamma i=\gamma s i$ ). Thus $\gamma$ has only nonnegative entries.

If $a, b, c, d \geqslant 0$ and $a d-b c=1$, then $c / a$ and $d / b$ are both $\leqslant 1$ or both $\geqslant 1$ (since open intervals between consecutive Farey fractions are either nonintersecting or one contains the other). Since $\gamma i \in \mathbf{I} \Longleftrightarrow a^{2}+b^{2}<c^{2}+d^{2}$, it follows that both $a / c$ and $b / d$ are $\leqslant 1$ for $\gamma i \in \mathbf{I}$. We conclude that among the eight matrices $\pm \gamma$, $\pm \gamma s, \pm \tilde{\gamma}, \pm \tilde{\gamma} s$, which have symmetric angles (see Figure 2), the one for which $\gamma i$ is in quadrant $\mathbf{I}$ can be chosen such that

$$
a, b, c, d \geqslant 0 \quad \text { and } \quad 0 \leqslant \frac{b}{d}<\frac{a}{c} \leqslant 1 .
$$

The set of such matrices $\gamma$ is denoted $\Gamma_{\mathbf{I}}$.

Consider the subset $\mathfrak{R}_{Q}$ of $\Gamma_{\mathbf{I}}$ consisting of matrices with entries at most $Q$ :

$$
\mathfrak{R}_{Q}:=\left\{\left(\begin{array}{cc}
p^{\prime} & p \\
q^{\prime} & q
\end{array}\right) \in \Gamma: 0 \leqslant p, p^{\prime}, q, q^{\prime} \leqslant Q, \frac{p}{q}<\frac{p^{\prime}}{q^{\prime}} \leqslant 1\right\}
$$

and its subset $\widetilde{\mathfrak{R}}_{Q}$ consisting of those $\gamma$ with $\|\gamma\| \leqslant Q$. The cardinality $B_{Q}$ of $\widetilde{\mathfrak{R}}_{Q}$ is estimated in Corollary 8 as $B_{Q} \sim 3 Q^{2} / 8$, in agreement with formula (58) in [Sarnak 2007] for the number of reciprocal geodesics of length at most $x=Q^{2}$.

Let $\mathscr{F}_{Q}$ be the set of Farey fractions $p / q$ with $0 \leqslant p \leqslant q \leqslant Q$ and $(p, q)=1$. The Farey tessellation (Figure 3 ) consists of semicircles on the upper half-plane

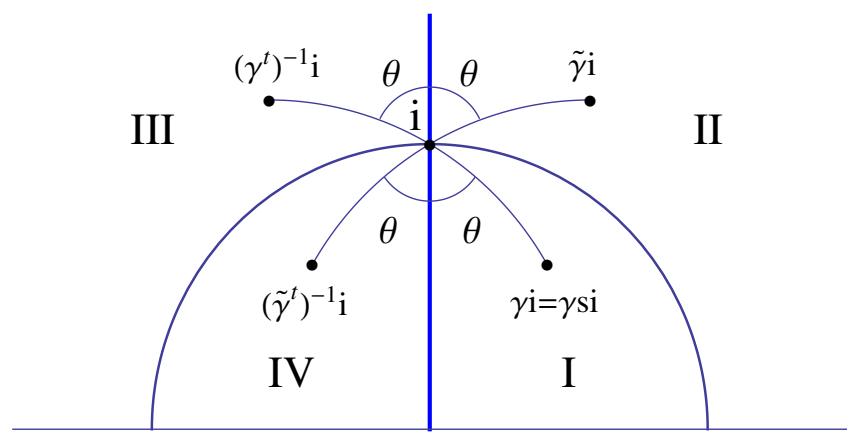

Figure 2. Two symmetric geodesics through $i$. 


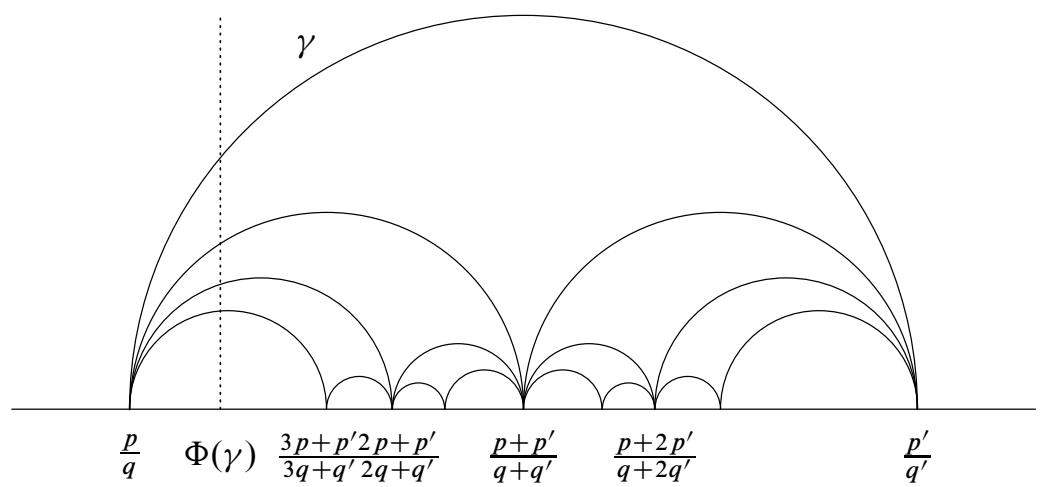

Figure 3. The Farey tessellation.

connecting Farey fractions $0 \leqslant p / q<p^{\prime} / q^{\prime} \leqslant 1$ with $p^{\prime} q-p q^{\prime}=1$. We associate to matrices $\gamma \in \mathfrak{R}_{Q}$ with entries as above the arc in the Farey tessellation connecting $p / q$ and $p^{\prime} / q^{\prime}$, and conclude that

$$
\# \Re_{Q}=2 \# \mathscr{F}_{Q}-3=\frac{Q^{2}}{\zeta(2)}+O(Q \ln Q) .
$$

\section{The coincidence of the pair correlations of $\Phi$ and $\Psi$}

In this section we show that the limiting pair correlations of the sets $\{\Psi(\gamma)\}$ and $\{\Phi(\gamma)\}$ ordered by $\|\gamma\| \rightarrow \infty$ do coincide. The proof uses properties of the Farey tessellation, via the correspondence between elements of $\mathfrak{R}_{Q}$ and arcs in the Farey tessellation defined at the end of Section 3.

For $\gamma=\left(\begin{array}{c}p^{\prime} p \\ q^{\prime}\end{array}\right)$, set $\gamma_{-}=p / q, \gamma_{+}=p^{\prime} / q^{\prime}$. From (3-1), (3-3), and the inequalities $X_{\gamma}<Z_{\gamma}<Y_{\gamma}, 2 Y_{\gamma}>T_{\gamma}$ and $\epsilon_{\gamma}<1 / T_{\gamma}$, we have:

$$
\begin{gathered}
\Psi(\gamma)-\Phi(\gamma)=\frac{Z_{\gamma}}{Y_{\gamma}\left(\epsilon_{\gamma}^{-1} Y_{\gamma}-1\right)} \ll \frac{1}{\|\gamma\|^{4}}, \\
\gamma_{-}<\Phi(\gamma)<\Psi(\gamma)<\gamma_{+} .
\end{gathered}
$$

Denote by $\mathscr{R}_{Q}^{\Psi}(\xi)\left(\right.$ resp. $\left.\mathscr{R}_{Q}^{\Phi}(\xi)\right)$ the number of pairs $\left(\gamma, \gamma^{\prime}\right) \in \widetilde{\mathfrak{R}}_{Q}^{2}, \gamma \neq \gamma^{\prime}$, such that $0 \leqslant \Psi(\gamma)-\Psi\left(\gamma^{\prime}\right) \leqslant \xi / Q^{2}$ (resp. $\left.0 \leqslant \Phi(\gamma)-\Phi\left(\gamma^{\prime}\right) \leqslant \xi / Q^{2}\right)$. For fixed $\beta \in\left(\frac{2}{3}, 1\right)$, consider also

$$
\mathcal{N}_{Q, \xi, \beta}^{\Psi}:=\#\left\{\left(\gamma, \gamma^{\prime}\right) \in \widetilde{\mathfrak{R}}_{Q}^{2}: Q^{2}\left|\Psi(\gamma)-\Psi\left(\gamma^{\prime}\right)\right| \leqslant \xi,\|\gamma\| \leqslant Q^{\beta}\right\},
$$

and the similarly defined $\mathcal{N}_{Q, \xi, \beta}^{\Phi}$. The trivial inequality

$\mathscr{R}_{Q}^{\Phi}(\xi) \leqslant 2 \mathcal{N}_{Q, \xi, \beta}^{\Phi}$

$$
+\#\left\{\left(\gamma, \gamma^{\prime}\right) \in \widetilde{\mathfrak{R}}_{Q}^{2}: \gamma \neq \gamma^{\prime}, Q^{2}\left|\Phi(\gamma)-\Phi\left(\gamma^{\prime}\right)\right| \leqslant \xi,\|\gamma\|,\left\|\gamma^{\prime}\right\| \geqslant Q^{\beta}\right\}
$$


and the estimate in (4-1) show that there exists a universal constant $\kappa>0$ such that $\mathscr{R}_{Q}^{\Phi}(\xi) \leqslant 2 \mathcal{N}_{Q, \xi, \beta}^{\Phi}$

$$
+\#\left\{\left(\gamma, \gamma^{\prime}\right) \in \widetilde{\mathfrak{R}}_{Q}^{2}: \gamma \neq \gamma^{\prime},-2 \kappa Q^{-4 \beta} \leqslant \Psi(\gamma)-\Psi\left(\gamma^{\prime}\right) \leqslant \xi Q^{-2}+2 \kappa Q^{-4 \beta}\right\},
$$

showing that

$$
\mathscr{R}_{Q}^{\Phi}(\xi) \leqslant 2 \mathcal{N}_{Q, \xi, \beta}^{\Phi}+\mathscr{R}_{Q}^{\Psi}\left(2 \kappa Q^{2-4 \beta}\right)+\mathscr{R}_{Q}^{\Psi}\left(\xi+2 \kappa Q^{2-4 \beta}\right) .
$$

In a similar way we show that

$$
\mathscr{R}_{Q}^{\Psi}(\xi) \leqslant 2 \mathcal{N}_{Q, \xi, \beta}^{\Psi}+\mathscr{R}_{Q}^{\Phi}\left(2 \kappa Q^{2-4 \beta}\right)+\mathscr{R}_{Q}^{\Phi}\left(\xi+2 \kappa Q^{2-4 \beta}\right) .
$$

We first prove that $\mathcal{N}_{Q, \xi, \beta}^{\Phi}$ and $\mathcal{N}_{Q, \xi, \beta}^{\Psi}$ are much smaller than $Q^{2}$. For this goal and for later use, it is important to divide pairs $\left(\gamma, \gamma^{\prime}\right) \in \mathfrak{R}_{O}^{2}$ in three cases, depending on the relative position of their associated arcs in the Farey tessellation (it is well known that two arcs in the Farey tessellation are nonintersecting):

(i) The arcs corresponding to $\gamma$ and $\gamma^{\prime}$ are exterior, i.e., $\gamma_{+} \leqslant \gamma_{-}^{\prime}$ or $\gamma_{+}^{\prime} \leqslant \gamma_{-}$.

(ii) $\gamma^{\prime} \lesssim \gamma$, i.e., $\gamma_{-} \leqslant \gamma_{-}^{\prime}<\gamma_{+}^{\prime} \leqslant \gamma_{+}$.

(iii) $\gamma \lesssim \gamma^{\prime}$, i.e., $\gamma_{-}^{\prime} \leqslant \gamma_{-}<\gamma_{+} \leqslant \gamma_{+}^{\prime}$.

\section{Proposition 3. $\quad \mathcal{N}_{Q, \xi, \beta}^{\Phi} \ll Q^{1+\beta} \ln Q \quad$ and $\quad \mathcal{N}_{Q, \xi, \beta}^{\Psi} \ll Q^{1+\beta} \ln Q$.}

Proof. $\mathcal{N}_{Q, \xi, \beta}^{\Phi}$ and $\mathcal{N}_{Q, \xi, \beta}^{\Psi}$ are increasing as an effect of enlarging $\widetilde{\mathfrak{R}}_{Q}$ to $\mathfrak{R}_{Q}$, so for this proof we will replace $\widetilde{\mathfrak{R}}_{Q}$ by $\mathfrak{R}_{Q}$. We only consider $\mathcal{N}_{Q, \xi, \beta}^{\Phi}$ here. The proof for the bound on $\mathcal{N}_{Q, \xi, \beta}^{\Psi}$ is identical. Both rely on (4-1) and (4-2).

Set $K=[\xi]+1$. From (4-2) and the fact that $\left|r^{\prime}-r\right| \geqslant 1 / Q^{2}$ for all $r, r^{\prime} \in \mathscr{F}_{Q}$ such that $r \neq r^{\prime}$, it follows that

$$
\#\left(\mathscr{F}_{Q} \cap\left[\gamma_{+}, \gamma_{-}^{\prime}\right]\right) \leqslant K+1
$$

if $\gamma_{+} \leqslant \gamma_{-}^{\prime}$ and $\left|\Phi\left(\gamma^{\prime}\right)-\Phi(\gamma)\right| \leqslant \xi / Q^{2}$. In particular, $\gamma_{-}^{\prime}=\gamma_{+}$when $0<\xi<1$.

We now consider the three cases listed before the statement of the proposition:

(i) The arcs corresponding to $\gamma$ and $\gamma^{\prime}$ are exterior. Without loss of generality, assume that $\gamma_{+} \leqslant \gamma_{-}^{\prime}$. If $i$ is such that $\gamma_{+}=\gamma_{i}$, the $i$-th element of $\mathscr{F}_{Q}$, then

$$
\gamma_{-}^{\prime}=\gamma_{i+r}=\frac{p_{i+r}}{q_{i+r}}
$$

for some $r$ with $0 \leqslant r<K$. The equality $p_{+}^{\prime} q_{-}^{\prime}-p_{-}^{\prime} q_{+}^{\prime}=1$ shows that if $\gamma_{-}^{\prime}=p_{-}^{\prime} / q_{-}^{\prime}$ is fixed, then $q_{+}^{\prime}$ (and therefore $\gamma_{+}^{\prime}=p_{+}^{\prime} / q_{+}^{\prime}$ ) is uniquely determined in intervals of length at most $q_{-}^{\prime}$. Since $q_{ \pm}^{\prime} \leqslant Q$, it follows that the number of choices for $q_{+}^{\prime}$ is actually at most $\left(Q / q_{-}^{\prime}\right)+1=\left(Q / q_{i+r}\right)+1$. 
When $0<\xi<1$ one must have $\gamma_{-}^{\prime}=\gamma_{+}$. Knowing $q_{-}$and $q_{+}$would uniquely determine the matrix $\gamma$. Then there will be at most $\left(Q / q_{+}\right)+1$ choices for $\gamma^{\prime}$, so the total contribution of this case to $\mathcal{N}_{Q, \xi, \beta}^{\Phi}$ is at most

$$
\sum_{1 \leqslant q_{-} \leqslant Q^{\beta}} \sum_{1 \leqslant q_{+} \leqslant Q^{\beta}}\left(\frac{Q}{q_{+}}+1\right) \ll Q^{1+\beta} \ln Q .
$$

When $\xi \geqslant 1$ denote by $q_{i}, q_{i+1}, \ldots, q_{i+K}$ the denominators of $\gamma_{i}, \gamma_{i+1}, \ldots, \gamma_{i+K}$. Since $q_{i}<Q^{\beta}$, we have

$$
\gamma_{i+K}-\gamma_{i} \leqslant \frac{K}{Q} \leqslant \frac{1}{Q^{\beta}} \leqslant \frac{1}{q_{i}} \leqslant 1-\gamma_{i},
$$

showing that $i+K<\# \mathscr{F}_{Q}$ so long as $Q \gg \xi 1$. As noticed in [Hall and Tenenbaum 1984],

$$
q_{j+2}=\left[\frac{Q+q_{j}}{q_{j+1}}\right] q_{j+1}-q_{j} .
$$

As in [Boca et al. 2001], consider

$$
\kappa(x, y):=\left[\frac{1+x}{y}\right] \text { and } \mathscr{T}_{k}=\left\{(x, y) \in(0,1]^{2}: x+y>1, \kappa(x, y)=k\right\} .
$$

Let $Q$ be large enough so that $\delta_{0}:=Q^{\beta-1}<1 /(2 K+3)$. Then $q_{i} / Q<\delta_{0}$, and it is plain (see also [ibid.]) that

and

$$
\frac{q_{i+1}}{Q}>1-\delta_{0}, \quad \kappa\left(\frac{q_{i}}{Q}, \frac{q_{i+1}}{Q}\right)=1,
$$

$$
\kappa\left(\frac{q_{i+1}}{Q}, \frac{q_{i+2}}{Q}\right)=\cdots=\kappa\left(\frac{q_{i+K}}{Q}, \frac{q_{i+K+1}}{Q}\right)=2,
$$

because $q_{i+1}, q_{i+2}, \ldots, q_{i+K+1}$ must form an arithmetic progression. Hence

$$
\left(\frac{q_{i}}{Q}, \frac{q_{i+1}}{Q}\right) \in \mathscr{T}_{1} \quad \text { and } \quad\left(\frac{q_{i+1}}{Q}, \frac{q_{i+2}}{Q}\right), \ldots,\left(\frac{q_{i+K}}{Q}, \frac{q_{i+K+1}}{Q}\right) \in \mathscr{T}_{2},
$$

showing in particular that $\min \left\{q_{i+1}, \ldots, q_{i+K}\right\}>Q / 3$. Therefore, we find that $\max \left\{Q / q_{i+1}, \ldots, Q / q_{i+K}\right\}<3$, and the contribution of this case to $\mathcal{N}_{Q, \xi, \beta}^{\Phi}$ is at most

$$
\sum_{1 \leqslant q_{-} \leqslant Q^{\beta}} \sum_{1 \leqslant q_{+} \leqslant Q^{\beta}} 4 K \ll \xi Q^{2 \beta}
$$

(ii) $\gamma^{\prime} \lesssim \gamma$. Let $i$ be the unique index for which $\gamma_{i}<\Phi(\gamma)<\gamma_{i+1}$ with $\gamma_{i}<\gamma_{i+1}$ successive elements in $\mathscr{F}_{Q}$. Since $\left|\Phi\left(\gamma^{\prime}\right)-\Phi(\gamma)\right| \leqslant \xi / Q^{2}$, either $\gamma_{-}^{\prime}<\Phi(\gamma)<\gamma_{+}^{\prime}$ or there exists $0 \leqslant r \leqslant K$ with $\gamma_{+}^{\prime}=\gamma_{i-r}$ or with $\gamma_{-}^{\prime}=\gamma_{i+r}$. In both situations the arc corresponding to the matrix $\gamma^{\prime}$ will cross at least one of the vertical lines above $\gamma_{i-K}, \ldots, \gamma_{i}, \gamma_{i+1}, \ldots, \gamma_{i+K}$. A glance at the Farey tessellation provides an upper bound for this number $N_{\gamma, K}$ of $\operatorname{arcs} \gamma^{\prime} \in \mathfrak{R}_{Q}$. Actually, one sees that the set $\mathscr{C}_{\gamma, L}$ consisting of $2+2^{2}+\cdots+2^{L}$ arcs obtained from $\gamma$ by iterating the 
mediant construction $L=\left[Q / \min \left\{q_{-}, q_{+}\right\}\right]+1$ times $\left(\gamma\right.$ is not enclosed in $\left.\mathscr{C}_{\gamma, L}\right)$ contains the set $\left\{\gamma^{\prime} \in \mathfrak{R}_{Q}: \gamma^{\prime} \lesssim \gamma, \gamma^{\prime} \neq \gamma\right\}$. The former set contains at most $L$ arcs that are intersected by each vertical direction, and so $N_{\gamma, K} \leqslant(2 K+1) L$. Therefore, the contribution of this case to $\mathcal{N}_{Q, \xi, \beta}^{\Phi}$ is (first choose $\gamma$, then $\gamma^{\prime}$ ) at most

$$
\sum_{1 \leqslant q \leqslant Q^{\beta}} \sum_{1 \leqslant q^{\prime} \leqslant Q^{\beta}}(2 K+1)\left(\frac{Q}{\min \left\{q, q^{\prime}\right\}}+1\right) \ll_{\xi} Q^{1+\beta} \ln Q .
$$

(iii) $\gamma \lesssim \gamma^{\prime}$. We necessarily have $\gamma=\gamma^{\prime} M$, with $M \in \mathfrak{S}$. In particular, this yields $\gamma_{ \pm}^{\prime} \in \mathscr{F}_{Q^{\beta}}$. Considering the subtessellation defined only by arcs connecting points from $\mathscr{F}_{Q^{\beta}}$, one sees that the number of arcs intersected by a vertical line $x=\alpha$ with

$$
\gamma_{-}=\frac{p}{q}<\alpha<\gamma_{+}=\frac{p^{\prime}}{q^{\prime}}, \quad \text { where } \gamma=\left(\gamma_{-}, \gamma_{+}\right) \in \mathscr{F}_{Q^{\beta}},
$$

is equal to $s\left(q, q^{\prime}\right)$, the sum of digits in the continued fraction expansion of $q / q^{\prime}<1$ when $q<q^{\prime}$, and respectively to $s\left(q^{\prime}, q\right)$ when $q^{\prime}<q$. A result from [Yao and Knuth 1975] yields in particular that

$$
\sum_{0<q<q^{\prime} \leqslant Q^{\beta}} s\left(q, q^{\prime}\right) \ll Q^{2 \beta} \ln ^{2} Q,
$$

and therefore

$$
\#\left\{\left(\gamma, \gamma^{\prime}\right) \in \mathfrak{R}_{Q^{\beta}}^{2}: \gamma \lesssim \gamma^{\prime}\right\} \leqslant 1+2 \sum_{0<q<q^{\prime} \leqslant Q^{\beta}} s\left(q, q^{\prime}\right) \ll Q^{2 \beta} \ln ^{2} Q .
$$

This completes the proof of the proposition.

Proposition 3 and inequalities (4-3) and (4-4) imply:

Corollary 4. For each $\beta \in\left(\frac{2}{3}, 1\right)$,

$$
\mathscr{R}_{Q}^{\Psi}(\xi)=\mathscr{R}_{Q}^{\Phi}\left(\xi+O_{\xi}\left(Q^{2-3 \beta}\right)\right)+\mathscr{R}_{Q}^{\Phi}\left(O_{\xi}\left(Q^{2-3 \beta}\right)\right)+O_{\xi}\left(Q^{1+\beta} \ln Q\right) .
$$

\section{A decomposition of the pair correlation of $\{\Phi(\gamma)\}$}

To estimate $\mathscr{R}_{Q}^{\Phi}(\xi)$, recall the correspondence between elements of $\Re_{Q}$ and arcs in the Farey tessellation from the end of Section 3. We consider the following two possibilities for the arcs associated to a pair $\left(\gamma, \gamma^{\prime}\right) \in \widetilde{\mathfrak{R}}_{Q}^{2}$ :

(i) One of the arcs corresponding to $\gamma$ and $\gamma^{\prime}$ contains the other.

(ii) The arcs corresponding to $\gamma$ and $\gamma^{\prime}$ are exterior (possibly tangent).

Denoting by $R_{Q}^{\text {ก }}(\xi)$ and $R_{Q}^{\cap \cap}(\xi)$ the number of pairs in each case, we have

$$
\mathscr{R}_{Q}^{\Phi}(\xi)=R_{Q}^{\bigcap}(\xi)+R_{Q}^{\cap \cap}(\xi) .
$$




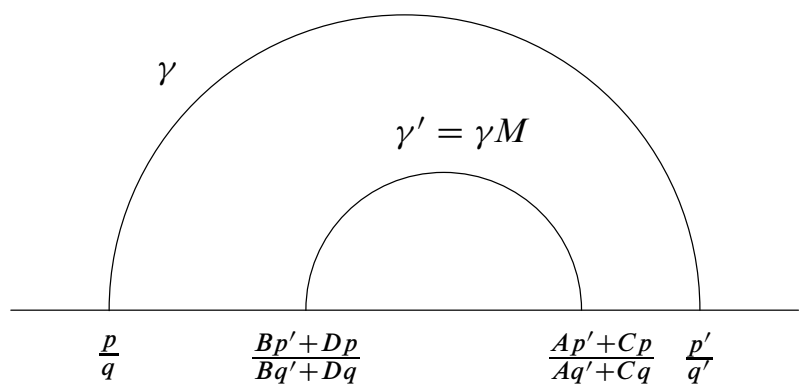

Figure 4. The case $\gamma^{\prime} \lesssim \gamma$.

5.1. One of the arcs contains the other. In this case we have either $\gamma=\gamma^{\prime} M$ or $\gamma^{\prime}=\gamma M$ with $M \in \mathfrak{S}$ (see also Figure 4). For each $M \in \Gamma$ define

$$
\Xi_{M}(x, y)=\frac{x y\left(Y_{M}-X_{M}\right)+\left(x^{2}-y^{2}\right) Z_{M}}{\left(x^{2}+y^{2}\right)\left(x^{2} X_{M}+y^{2} Y_{M}+2 x y Z_{M}\right)},
$$

where $X_{M}, Y_{M}, Z_{M}$ are defined in (3-1). For $\gamma=\left(\begin{array}{c}p^{\prime} \\ q^{\prime}\end{array}\right)$, a direct calculation shows

$$
\Phi(\gamma)-\Phi(\gamma M)=\Xi_{M}\left(q^{\prime}, q\right) .
$$

Two remarks are in order now. First, notice that $X_{M} \neq Y_{M}$ for any $M \in \mathfrak{S}$ because of (3-2) and since $X_{M}, Y_{M}, Z_{M} \geqslant 1$. Secondly, we also have

$$
\Phi(\gamma) \neq \Phi(\gamma M) .
$$

Suppose, ad absurdum, that $\Phi(\gamma)=\Phi(\gamma M)$. Then (5-2) and (5-1) yield

$$
\frac{2 Z_{M}}{Y_{M}-X_{M}}=\frac{2 q q^{\prime}}{q^{2}-q^{\prime 2}}
$$

that is, $\tan \theta_{M}=\tan 2 \theta$, where $\theta=\tan ^{-1}\left(q^{\prime} / q\right) \in(0, \pi)$ and $\theta_{M} \in(0, \pi)$ because $Z_{M}>0$. This gives

$$
\frac{X_{M}-\epsilon_{M}}{Z_{M}}=\tan \left(\frac{\theta_{M}}{2}\right)=\tan \theta \in \mathbb{Q},
$$

hence $\sqrt{\left(X_{M}+Y_{M}\right)^{2}-4}=X_{M}+Y_{M}-2 \epsilon_{M} \in \mathbb{Q}$, which is not possible because $X_{M}+Y_{M} \geqslant 3$.

From (5-2) and (5-3) we now infer:

Lemma 5. Using the notation introduced before Proposition 3, the number of pairs $\left(\gamma, \gamma^{\prime}\right) \in \widetilde{\mathfrak{R}}_{Q}^{2}, \gamma \neq \gamma^{\prime}$, with $0 \leqslant \Phi(\gamma)-\Phi\left(\gamma^{\prime}\right) \leqslant \xi / Q^{2}$ and $\gamma \lesssim \gamma^{\prime}$ or $\gamma^{\prime} \lesssim \gamma$, is given by

$$
R_{Q}^{\cap}(\xi)=\#\left\{(\gamma, \gamma M) \in \widetilde{\mathfrak{R}}_{Q}^{2}: \gamma=\left(\begin{array}{cc}
p^{\prime} & p \\
q^{\prime} & q
\end{array}\right), M \in \mathfrak{S},\left|\Xi_{M}\left(q^{\prime}, q\right)\right| \leqslant \frac{\xi}{Q^{2}}\right\} .
$$




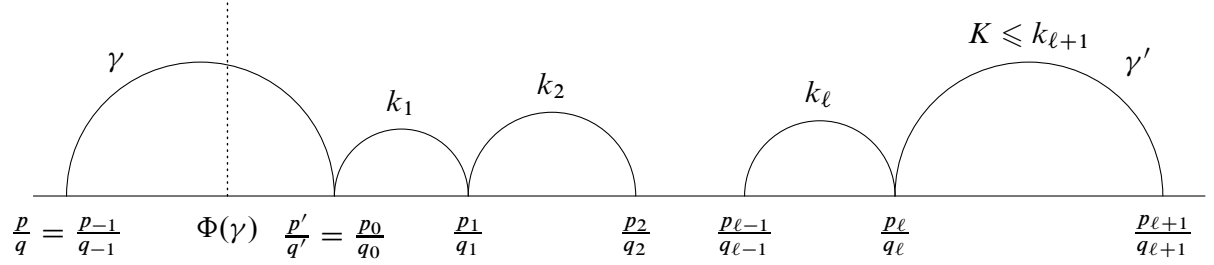

Figure 5. The case where $\gamma$ and $\gamma^{\prime}$ are exterior.

5.2. Exterior arcs. In this case we have $\gamma, \gamma^{\prime} \in \widetilde{\mathfrak{R}}_{Q}, \gamma_{-}^{\prime} \geqslant \gamma_{+}$. Let $\ell \geqslant 0$ be the number of Farey arcs in $\mathscr{F}_{Q}$ connecting the arcs corresponding to $\gamma, \gamma^{\prime}$ (see Figure 5). In other words, writing $\gamma=\left(\begin{array}{cc}p^{\prime} & p \\ q^{\prime} & q\end{array}\right)$ and $\gamma^{\prime}=\left(\begin{array}{ll}p_{\ell+1} & p_{\ell} \\ q_{\ell+1} & q_{\ell}\end{array}\right)$, we have that $p_{0} / q_{0}:=p^{\prime} / q^{\prime}, p_{1} / q_{1}, \ldots, p_{\ell} / q_{\ell}$ are consecutive elements in $\mathscr{F}_{Q}$. Setting also $p_{-1} / q_{-1}:=p / q$, it follows that $q_{i}=k_{i} q_{i-1}-q_{i-2}$, where $k_{i} \in \mathbb{N}, i=1, \ldots, \ell$, and

$$
k_{i}=\left[\frac{Q+q_{i-2}}{q_{i-1}}\right] \text { for } 2 \leqslant i \leqslant \ell .
$$

The fractions $p_{\ell} / q_{\ell}$ and $p_{\ell+1} / q_{\ell+1}$ are not necessarily consecutive in $\mathscr{F}_{Q}$, but we have $q_{\ell+1}=K q_{\ell}-q_{\ell-1}$,

$$
K \leqslant k_{\ell+1}=\left[\frac{Q+q_{\ell}}{q_{\ell+1}}\right] .
$$

It follows that $\gamma^{\prime}=\gamma M$ with

$$
M=\left(\begin{array}{cc}
k_{1} & 1 \\
-1 & 0
\end{array}\right) \cdots\left(\begin{array}{cc}
k_{\ell} & 1 \\
-1 & 0
\end{array}\right)\left(\begin{array}{cc}
K & 1 \\
-1 & 0
\end{array}\right) .
$$

We have $\ell<\xi$ because

It is also plain to see that

$$
\Phi\left(\gamma^{\prime}\right)-\Phi(\gamma)>\sum_{i=1}^{\ell} \frac{1}{q_{i-1} q_{i}} \geqslant \frac{\ell}{Q^{2}} .
$$

$$
\frac{p^{\prime}}{q^{\prime}}-\Phi(\gamma)=\frac{q}{q^{\prime}\left(q^{2}+q^{\prime 2}\right)}, \quad \Phi\left(\gamma^{\prime}\right)-\frac{p_{\ell}}{q_{\ell}}=\frac{q_{\ell+1}}{q_{\ell}\left(q_{\ell}^{2}+q_{\ell+1}^{2}\right)} .
$$

The last equality in (5-4) and $q_{\ell}^{2}+q_{\ell+1}^{2} \leqslant Q^{2}$ yield, for $\ell \geqslant 1$,

$$
\begin{aligned}
\frac{\xi}{Q^{2}} \geqslant \Phi\left(\gamma^{\prime}\right)-\Phi(\gamma) \geqslant \frac{1}{q_{\ell-1} q_{\ell}} & +\frac{q_{\ell+1}}{q_{\ell}\left(q_{\ell}^{2}+q_{\ell+1}^{2}\right)} \\
& \geqslant \frac{1}{q_{\ell-1} q_{\ell}}+\frac{K q_{\ell}-q_{\ell-1}}{q_{\ell} Q^{2}}=\frac{K}{Q^{2}}+\frac{Q^{2}-q_{\ell-1}^{2}}{q_{\ell-1} q_{\ell} Q^{2}} \geqslant \frac{K}{Q^{2}},
\end{aligned}
$$

while if $\ell=0$ we have

$$
\Phi\left(\gamma^{\prime}\right)-\Phi(\gamma)=\frac{K\left(q^{\prime 2}+q q_{1}\right)}{\left(q^{2}+q^{\prime 2}\right)\left(q^{\prime 2}+q_{1}^{2}\right)} \geqslant \frac{K}{Q^{2}},
$$


showing that $K<\xi$. Notice also that (5-4) yields

$$
\Phi\left(\gamma^{\prime}\right)-\Phi(\gamma)=\frac{q}{q^{\prime}\left(q^{2}+q^{\prime 2}\right)}+\sum_{i=1}^{\ell} \frac{1}{q_{i-1} q_{i}}+\frac{q_{\ell+1}}{q_{\ell}\left(q_{\ell}^{2}+q_{\ell+1}^{2}\right)} .
$$

Let $\mathscr{T}=\left\{(x, y) \in(0,1]^{2}: x+y>1\right\}$ and consider the map

$$
T:(0,1]^{2} \rightarrow \mathscr{T}, \quad T(x, y)=\left(y,\left[\frac{1+x}{y}\right] y-x\right),
$$

whose restriction to $\mathscr{T}$ is bijective and area-preserving [Boca et al. 2001]. Consider the iterates $T^{i}=\left(L_{i-1}, L_{i}\right)$ and the functions $K_{i}=\left[\left(1+L_{i-2}\right) / L_{i-1}\right]$ if $i=1, \ldots, \ell$, $K_{\ell+1}=K$, and $L_{\ell+1}=K L_{\ell}-L_{\ell-1}$. One has:

- $L_{-1}(x, y)=x$ and $L_{0}(x, y)=y$ for $(x, y) \in(0,1]^{2}$;

- $0<L_{i}(x, y) \leqslant 1$ for $i \geqslant 0$ and $(x, y) \in \mathscr{T}$;

- $L_{i-1}(x, y)+L_{i}(x, y)>1$ for $i=1, \ldots, \ell$ and $(x, y) \in \mathscr{T}$

- $L_{i}(x, y)=K_{i}(x, y) L_{i-1}(x, y)-L_{i-2}(x, y)$

$$
\text { for } i=1, \ldots, \ell+1 \text { and }(x, y) \in \mathscr{T} \text {; }
$$

- $\left(q_{i-1}, q_{i}\right)=Q T^{i}\left(\frac{q}{Q}, \frac{q^{\prime}}{Q}\right)$

$$
=\left(Q L_{i-1}\left(\frac{q}{Q}, \frac{q^{\prime}}{Q}\right), Q L_{i}\left(\frac{q}{Q}, \frac{q^{\prime}}{Q}\right)\right) \text { for } i=0,1, \ldots, \ell ;
$$

- $q_{\ell+1}=K q_{\ell}-q_{\ell-1}=Q\left(K L_{\ell}\left(\frac{q}{Q}, \frac{q^{\prime}}{Q}\right)-L_{\ell-1}\left(\frac{q}{Q}, \frac{q^{\prime}}{Q}\right)\right)$.

Define also the function $\Upsilon_{\ell, K}:(0,1]^{2} \rightarrow(0, \infty)$ by

$$
\Upsilon_{\ell, K}=\frac{L_{-1}}{L_{0}\left(L_{-1}^{2}+L_{0}^{2}\right)}+\sum_{i=1}^{\ell} \frac{1}{L_{i-1} L_{i}}+\frac{L_{\ell+1}}{L_{\ell}\left(L_{\ell}^{2}+L_{\ell+1}^{2}\right)} .
$$

We have proved the following statement:

Lemma 6. The number $R_{Q}^{\cap \cap}(\xi)$ of pairs $\left(\gamma, \gamma^{\prime}\right)$ of exterior (possibly tangent) arcs in $\widetilde{\mathfrak{R}}_{Q}$ for which $0<\Phi\left(\gamma^{\prime}\right)-\Phi(\gamma) \leqslant \xi / Q^{2}$ is given by

$$
R_{Q}^{\cap \cap}(\xi)=\sum_{\substack{\ell \in[0, \xi) \\ K \in[1, \xi)}} d_{\ell K}
$$

where the sums are over integers in the given intervals, $d_{\ell K}$ is the number of matrices

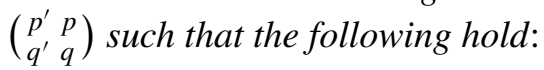


1016Florin P. Boca, Vicentiu Pasol, Alexandru A. Popa and Alexandru Zaharescu

$$
\begin{gathered}
0 \leqslant p \leqslant q, \quad 0 \leqslant p^{\prime} \leqslant q^{\prime}, \quad p^{\prime} q-p q^{\prime}=1, \\
p^{2}+p^{\prime 2}+q^{2}+q^{\prime 2} \leqslant Q^{2}, \quad 0<K q_{\ell}-q_{\ell-1} \leqslant Q, \\
p_{\ell}^{2}+q_{\ell}^{2}+\left(K p_{\ell}-p_{\ell-1}\right)^{2}+\left(K q_{\ell}-q_{\ell-1}\right)^{2} \leqslant Q^{2}, \\
\Upsilon_{\ell, K}\left(q / Q, q^{\prime} / Q\right) \leqslant \xi,
\end{gathered}
$$

and $q_{-1}=q, q_{0}=q^{\prime}$

\section{A lattice point estimate}

Lemma 7. Suppose that $\Omega$ is a region in $\mathbb{R}^{2}$ of area $A(\Omega)$ and rectifiable boundary of length $\ell(\partial \Omega)$. For any integer $r$ with $(r, q)=1$ and $1 \leqslant L \leqslant q$, we have

$$
\mathcal{N}_{\Omega, q, r}:=\#\left\{(a, b) \in \Omega \cap \mathbb{Z}^{2}: a b \equiv r(\bmod q)\right\}=\frac{\varphi(q)}{q^{2}} A(\Omega)+\mathscr{E}_{\Omega, L, q},
$$

where, for each $\varepsilon>0$,

$$
\mathscr{\mathscr { E }}_{\Omega, L, q} \ll_{\varepsilon} \frac{q^{1 / 2+\varepsilon} A(\Omega)}{L^{2}}+\left(1+\frac{\ell(\partial \Omega)}{L}\right)\left(\frac{L^{2}}{q}+q^{1 / 2+\varepsilon}\right) .
$$

Proof. Replacing $\mathbb{Z}^{2}$ by $L \mathbb{Z}^{2}$ in the estimate

$$
\left\{(m, n) \in \mathbb{Z}^{2}:(m, m+1) \times(n, n+1) \cap \partial \Omega \neq \varnothing\right\} \ll 1+\ell(\partial \Omega),
$$

(for a proof see [Narkiewicz 1983, Theorem 5.9]) we find that the number of squares $S_{m, n}=[L m, L(m+1)] \times[L n, L(n+1)]$ such that $\stackrel{\circ}{S}, n_{m} \cap \partial \Omega$ is nonempty is $\ll 1+(1 / L) \ell(\partial \Omega)$. Therefore

$\#\left\{(m, n) \in \mathbb{Z}^{2}:(L m, L(m+1)) \times(L n, L(n+1)) \subseteq \Omega\right\}=\frac{A(\Omega)}{L^{2}}+O\left(1+\frac{\ell(\partial \Omega)}{L}\right)$.

Weil's estimates [1948] on Kloosterman sums, extended to composite moduli in [Hooley 1957] and [Estermann 1961], show that each such square contains $\left(\varphi(q) / q^{2}\right) L^{2}+O_{\varepsilon}\left(q^{1 / 2+\varepsilon}\right)$ pairs of integers $(a, b)$ with $a b \equiv r(\bmod q)$ (see, e.g., [Boca et al. 2000, Lemma 1.7] for details). Combining these two estimates, we find $\mathcal{N}_{\Omega, q, r}=\left(\frac{A(\Omega)}{L^{2}}+O\left(1+\frac{\ell(\partial \Omega)}{L}\right)\right)\left(\frac{\varphi(q)}{q^{2}} L^{2}+O\left(q^{1 / 2+\varepsilon}\right)\right)=\frac{\varphi(q)}{q^{2}} A(\Omega)+\mathscr{E}_{\Omega, q, L}$, as desired.

Corollary 8.

$$
\# \widetilde{\Re}_{Q}=\frac{3 Q^{2}}{8}+O_{\varepsilon}\left(Q^{11 / 6+\varepsilon}\right)
$$

Proof. Note first that one can substitute $p q^{\prime} / q$ for $p^{\prime}=\left(1+p q^{\prime}\right) / q$ in the definition of $\widetilde{\mathfrak{R}}_{Q}$, replacing the inequality $\|\gamma\|^{2} \leqslant Q^{2}$ by $\left(q^{2}+q^{\prime 2}\right)\left(q^{2}+p^{2}\right) \leqslant Q^{2} q^{2}$, without altering the error term. Applying Lemma 7 with

$$
\Omega_{q}=\left\{(u, v) \in[0, q] \times[0, Q]:\left(q^{2}+u^{2}\right)\left(q^{2}+v^{2}\right) \leqslant Q^{2} q^{2}\right\} \quad \text { and } \quad L=q^{5 / 6},
$$


and using $A\left(\Omega_{q}\right) \leqslant Q q$ and $\ell\left(\Omega_{q}\right) \leqslant 2(Q+q) \leqslant 4 Q$, we infer that

$$
\# \widetilde{\Re}_{Q}=\sum_{q=1}^{Q} \frac{\varphi(q)}{q} \cdot \frac{A\left(\Omega_{q}\right)}{q}+O_{\varepsilon}\left(Q^{11 / 6+\varepsilon}\right) .
$$

Standard Möbius summation (see, e.g., [Boca et al. 2000, Lemma 2.3]) applied to the decreasing function $h(q)=(1 / q) A\left(\Omega_{q}\right)$ with $\|h\|_{\infty} \leqslant Q$ and the change of variable $(q, u, v)=(Q x, Q x y, Q z)$ further yield

where

$$
\# \widetilde{\mathfrak{R}}_{Q}=\frac{Q^{2}}{\zeta(2)} \operatorname{Vol}(S)+O_{\varepsilon}\left(Q^{11 / 6+\varepsilon}\right)
$$

$$
S=\left\{(x, y, z) \in[0,1]^{3}:\left(1+y^{2}\right)\left(x^{2}+z^{2}\right) \leqslant 1\right\} .
$$

The substitution $y=\tan \theta$ yields

$$
\operatorname{Vol}(S)=\int_{0}^{\pi / 4} \frac{d \theta}{\cos ^{2} \theta} A\left(\left\{(x, z) \in[0,1]^{2}: x^{2}+z^{2} \leqslant \cos ^{2} \theta\right\}\right)=\frac{\pi^{2}}{16},
$$

completing the proof of the corollary.

The error bound in Corollary 8 can be improved using spectral methods (see Corollary 12.2 in [Iwaniec 2002]). We have given the proof since it is the prototype of applying Lemma 7 to the counting problems of the next section.

\section{Pair correlation of $\{\Phi(\gamma)\}$}

The main result of this section is Theorem 2, where we obtain explicit formulas for the pair correlation of the quantities $\{\Phi(\gamma)\}$ in terms of volumes of threedimensional bodies. The discussion is divided in two cases, as in Section 5.

7.1. One of the arcs contains the other. The formula for $R_{Q}^{\text {ก }}$ in Lemma 5 provides

$$
R_{Q}^{\cap}(\xi)=\sum_{M \in \mathfrak{S}} \mathcal{N}_{M, Q}(\xi),
$$

where $\mathcal{N}_{M, Q}(\xi)$ denotes the number of matrices $\gamma=\left(\begin{array}{c}p^{\prime} \\ q^{\prime} \\ q\end{array}\right)$ for which

$0 \leqslant p \leqslant q, \quad 0 \leqslant p^{\prime} \leqslant q^{\prime}, \quad p^{\prime} q-p q^{\prime}=1, \quad\left|\Xi_{M}\left(q^{\prime}, q\right)\right| \leqslant \frac{\xi}{Q^{2}}, \quad\|\gamma M\| \leqslant Q$.

The first goal is to replace in (7-2) the inequality $\|\gamma M\| \leqslant Q$ by a more tractable one. Taking $\gamma$ of the given form and substituting $p=\left(p^{\prime} q-1\right) / q^{\prime}$ we write, using the notation from (3-1): $\|\gamma M\|^{2}=$

$$
\left(\frac{p^{\prime 2}}{q^{\prime 2}}+1\right)\left(q^{\prime 2} X_{M}+q^{2} Y_{M}+2 q q^{\prime} Z_{M}\right)-\frac{\left(p^{\prime} q+p q^{\prime}\right) Y_{M}+2 p^{\prime} q^{\prime} Z_{M}}{q^{\prime 2}} .
$$


The quantity $\mathcal{N}_{M, Q}(\xi)$ can be conveniently related to $\tilde{\mathcal{N}}_{M, Q}(\xi)$, the number of integer triples $\left(q^{\prime}, q, p^{\prime}\right)$ such that

$$
\begin{gathered}
0<p^{\prime} \leqslant q^{\prime} \leqslant Q, \quad 0<q \leqslant Q, \quad p^{\prime} q \equiv 1\left(\bmod q^{\prime}\right), \\
\left|\Xi_{M}\left(q^{\prime}, q\right)\right| \leqslant \frac{\xi}{Q^{2}}, \quad Y_{\gamma M}=q^{\prime 2} X_{M}+q^{2} Y_{M}+2 q q^{\prime} Z_{M} \leqslant \frac{Q^{2} q^{\prime 2}}{p^{\prime 2}+q^{\prime 2}} .
\end{gathered}
$$

We next prove that, given $c_{0} \in\left(\frac{1}{2}, 1\right)$, for all $M \in \mathfrak{S}$ and $Q \geqslant 1$ with $Y_{M}<X_{M} \leqslant$ $Q^{2 c_{0}}$ and all $\xi>0$,

$$
\tilde{\mathcal{N}}_{M, Q}(\xi) \leqslant \mathcal{N}_{M, Q}(\xi) \leqslant \tilde{\mathcal{N}}_{M, Q\left(1+\sqrt{2} Q^{c_{0}-1}\right)}\left(\xi\left(1+\sqrt{2} Q^{c_{0}-1}\right)^{2}\right) .
$$

For the first inequality, note that if the integral triple $\left(q^{\prime}, q, p^{\prime}\right)$ satisfies (7-4) then by (7-3) we have

$$
\|\gamma M\|^{2} \leqslant \frac{p^{\prime 2}+q^{\prime 2}}{q^{\prime 2}} Y_{\gamma M} \leqslant Q^{2},
$$

and thus if we define $p:=\left(p^{\prime} q-1\right) / q^{\prime}$ then (7-2) holds. For the second inequality, take $\gamma$ as in (7-2). Using (7-3) we then have

$$
\frac{p^{\prime 2}+q^{\prime 2}}{q^{\prime 2}} Y_{\gamma M} \leqslant Q^{2}+\frac{\left(p^{\prime} q+p q^{\prime}\right) Y_{M}+2 p^{\prime} q^{\prime} Z_{M}}{q^{\prime 2}} \leqslant Q^{2}+2 q Y_{M}+2 Z_{M} .
$$

Using also that $Z_{M} \leqslant Q^{2 c_{0}}$ and $q Y_{M}=\sqrt{q^{2} Y_{M}} \sqrt{Y_{M}} \leqslant \sqrt{Y_{\gamma M}} \sqrt{Y_{M}} \leqslant Q^{1+c_{0}}$, we conclude that

$$
\frac{p^{\prime 2}+q^{\prime 2}}{q^{\prime 2}} Y_{\gamma M} \leqslant Q^{2}+2 Q^{1+c_{0}}+2 Q^{2 c_{0}} \leqslant Q^{2}\left(1+\sqrt{2} Q^{c_{0}-1}\right)^{2} .
$$

Also

$$
\left|\Xi_{M}\left(q^{\prime}, q\right)\right| \leqslant \frac{\xi}{Q^{2}}=\frac{\xi\left(1+\sqrt{2} Q^{c_{0}-1}\right)^{2}}{Q^{2}\left(1+\sqrt{2} Q^{c_{0}-1}\right)^{2}} .
$$

Hence $\left(q^{\prime}, q, p^{\prime}\right)$ satisfies (7-4) with the pair $(Q, \xi)$ replaced by $\left(Q+\sqrt{2} Q^{c_{0}}\right.$, $\left.\xi\left(1+\sqrt{2} Q^{c_{0}-1}\right)^{2}\right)$. This proves (7-5).

Next we show that $\mathcal{N}_{M, Q}(\xi)$ vanishes when $\max \left\{X_{M}, Y_{M}\right\} \geqslant Q^{2 c_{0}}$ and $Q$ is large enough.

Lemma 9. Let $c_{0} \in\left(\frac{1}{2}, 1\right)$. There exists $Q_{0}(\xi)$ such that whenever $M \in \mathfrak{S}$, $\max \left\{X_{M}, Y_{M}\right\} \geqslant Q^{2 c_{0}}$, and $Q \geqslant Q_{0}(\xi)$,

$$
\mathcal{N}_{M, Q}(\xi)=\tilde{\mathcal{N}}_{M, Q}(\xi)=0 .
$$

Proof. We show there are no coprime positive integer lattice points $\left(q^{\prime}, q\right)$ for which

$$
\left|\Xi_{M}\left(q^{\prime}, q\right)\right| \leqslant \frac{\xi}{Q^{2}}, \quad Y_{\gamma M}=q^{\prime 2} X_{M}+q^{2} Y_{M}+2 q q^{\prime} Z_{M} \leqslant Q^{2} .
$$


Noting from (7-3) that $Y_{\gamma M} \leqslant\|\gamma M\|^{2}$, this will ensure that $\mathcal{N}_{M, Q}(\xi)=0$. The equality $\tilde{\mathcal{N}}_{M, Q}(\xi)=0$ follows as well from (7-4).

Suppose $\left(q^{\prime}, q\right)$ is as in (7-6), write $q^{\prime} i+q=\left(q, q^{\prime}\right)=(r \cos \theta, r \sin \theta), \theta \in$ $(0, \pi / 2)$, and consider $(X, Y, Z)=\left(X_{M}, Y_{M}, Z_{M}\right), T=\|M\|^{2}=X+Y$, and $U_{M}=\operatorname{coth} d(i, M i)=T / \sqrt{T^{2}-4}$. Since

$$
\sin \theta_{M}=\frac{2 Z}{\sqrt{T^{2}-4}} \quad \text { and } \quad \cos \theta_{M}=\frac{Y-X}{\sqrt{T^{2}-4}},
$$

the inequalities in (7-6) can be described as

$$
\frac{1}{\xi} \cdot \frac{\left|\sin \left(\theta_{M}-2 \theta\right)\right|}{U_{M}+\cos \left(\theta_{M}-2 \theta\right)} \leqslant \frac{r^{2}}{Q^{2}} \leqslant \frac{2}{\left(U_{M}+\cos \left(\theta_{M}-2 \theta\right)\right) \sqrt{T^{2}-4}} .
$$

Denoting $\delta_{M}=\theta_{M} / 2-\theta$, from the first and last fraction in (7-7) we infer $\left|\sin 2 \delta_{M}\right| \ll 1 / T$. Therefore $\delta_{M}$ is close to 0 , or to $\pm \pi / 2$. When $\delta_{M}$ is close to 0 we have

$$
\left|\tan \delta_{M}\right| \ll\left|\delta_{M}\right| \ll\left|\sin 2 \delta_{M}\right| \ll \frac{1}{T} .
$$

When $\delta_{M}$ is close to $\pm \pi / 2$ we similarly have $\left|\delta_{M} \mp \pi / 2\right| \ll \frac{1}{T}$, which is seen to be impossible. Indeed, the inequality

$$
\frac{\left|\tan \delta_{M}\right|}{1+\frac{U_{M}-1}{1+\cos 2 \delta_{M}}}=\frac{\left|\sin 2 \delta_{M}\right|}{U_{M}+\cos 2 \delta_{M}} \leqslant \xi
$$

shows that it suffices to bound from above $\left(U_{M}-1\right) /\left(1+\cos 2 \delta_{M}\right)$, which would imply that $\left|\tan \delta_{M}\right| \ll \xi$, thus contradicting $\left|\delta_{M} \mp \frac{\pi}{2}\right| \ll 1 / T$. Since $Z$ is a positive integer, we have $\sin \theta_{M} \gg 1 / T$. Since $\cos \theta, \sin \theta>0$ and $\theta_{M} \in(0, \pi)$, we have

$$
\begin{aligned}
1+\cos 2 \delta_{M}=1+\cos \left(\theta_{M}-2 \theta\right) & \geqslant 1+\cos 2 \theta \cos \theta_{M} \\
& \geqslant 1-\left|\cos \theta_{M}\right|=1-\sqrt{1-\sin ^{2} \theta_{M}} \gg \frac{1}{T^{2}} .
\end{aligned}
$$

As $U_{M}-1 \ll 1 / T^{2}$, it follows that $\left(U_{M}-1\right) /\left(1+\cos 2 \delta_{M}\right) \ll 1$, a contradiction.

We have thus shown that $\left|\delta_{M}\right| \leqslant\left|\tan \delta_{M}\right| \ll 1 / T$; more precisely, there exists a function $\Theta_{0}(\xi)$, continuous in $\xi$, such that $\left|\delta_{M}\right| \leqslant \Theta_{0}(\xi) / T$.

Case I: $Y>X$. Then $0<\theta_{M} / 2<\pi / 4$ and $Z=\sqrt{X Y-1}<Y$. Since

$$
\left|\delta_{M}\right| \ll \frac{1}{T} \ll Q^{-2 c_{0}},
$$

one has $0<\theta<\pi / 3$ for large $Q$. Employing the formula $\tan \left(\theta_{M} / 2\right)=Z /\left(Y-\epsilon_{T}\right)$ with $\epsilon_{T}$ as in (3-1), we infer

$$
\left|\frac{A C+B D}{C^{2}+D^{2}-\epsilon_{T}}-\frac{q^{\prime}}{q}\right|=\left|\tan \delta_{M}\right| \cdot\left|1+\tan \theta \tan \frac{\theta_{M}}{2}\right| \ll \frac{1}{T} .
$$


Combining (7-8) with

$$
0<\frac{Z}{Y-\epsilon_{T}}-\frac{Z}{Y} \ll \frac{1}{T} \quad \text { and } \quad\left|\frac{Z}{Y}-\frac{A+B}{C+D}\right| \leqslant \frac{1}{C^{2}+D^{2}} \ll \frac{1}{T},
$$

we arrive at

$$
\left|\frac{A+B}{C+D}-\frac{q^{\prime}}{q}\right| \ll \frac{1}{T} \leqslant Q^{-2 c_{0}} .
$$

If nonzero, the left-hand side in (7-9) must be at least $1 / q(C+D)$. But

$$
q(C+D) \leqslant q \sqrt{2\left(C^{2}+D^{2}\right)} \leqslant Q \sqrt{2},
$$

and so $Q^{2 c_{0}} \ll Q$, a contradiction. The remaining case, in which $q=C+D$ and $q^{\prime}=A+B$, is not possible because $Q^{2 c_{0}} \leqslant(C+D)^{2}=q(C+D) \leqslant Q \sqrt{2}$.

Case II: $X>Y$. Then $\pi / 4<\theta_{M} / 2<\pi / 2$ and $Y \leqslant \sqrt{X Y-1}=Z$. As $\left|\delta_{M}\right| \ll Q^{-2 c_{0}}$, we must have $0<\pi / 2-\theta<\pi / 3$ for large values of $Q$. This time we have

$$
\begin{aligned}
\left|\frac{Y-\varepsilon_{T}}{Z}-\frac{q}{q^{\prime}}\right| & =\left|\tan \left(\frac{\pi}{2}-\frac{\theta_{M}}{2}\right)-\tan \left(\frac{\pi}{2}-\theta\right)\right| \\
& =\left|\tan \delta_{M}\right| \cdot\left|1+\tan \left(\frac{\pi}{2}-\frac{\theta_{M}}{2}\right) \tan \left(\frac{\pi}{2}-\theta\right)\right| \\
& \leqslant(1+\sqrt{3})\left|\tan \delta_{M}\right| \ll \frac{1}{T},
\end{aligned}
$$

which leads (since $D \geqslant C$ if and only if $B \geqslant A$ ) to

$$
\begin{aligned}
\left|\frac{C+D}{A+B}-\frac{q}{q^{\prime}}\right| & \ll \frac{1}{T}+\frac{\epsilon_{T}}{Z}+\left|\frac{Y}{Z}-\frac{C+D}{A+B}\right| \ll \frac{1}{T}+\frac{|D-C|}{(A+B)(A C+B D)} \\
& \leqslant \frac{1}{T}+\frac{1}{(A+B)^{2}} \leqslant \frac{1}{T}+\frac{1}{X} \ll \frac{1}{T} \ll Q^{-2 c_{0}} .
\end{aligned}
$$

As in Case I, this is not possible because $q^{\prime}(A+B) \leqslant q^{\prime} \sqrt{2 X} \leqslant Q \sqrt{2}$ and $(A+B)^{2} \geqslant Q^{2 c_{0}}$.

Our next goal is to apply Lemma 7, assuming $Y_{M}<X_{M} \ll Q^{2 c_{0}}$ and taking $r=1$, to the set $\Omega=\Omega_{M, q^{\prime}, \xi}$ of pairs $(u, v) \in(0, Q] \times\left(0, q^{\prime}\right]$ that satisfy

$$
\left|\Xi_{M}\left(q^{\prime}, u\right)\right| \leqslant \frac{\xi}{Q^{2}} \quad \text { and } \quad q^{\prime 2} X_{M}+u^{2} Y_{M}+2 u q^{\prime} Z_{M} \leqslant \frac{Q^{2} q^{\prime 2}}{v^{2}+q^{\prime 2}} .
$$

Lemma 10. There exist continuous functions $T_{0}(\xi)$ and $C(\xi)$ such that, for any matrix $M \in \mathfrak{S}$ with $Y_{M}<X_{M}$ and $T=\|M\|^{2}>T_{0}(\xi)$, the projection on the first coordinate of the set $\Omega_{M, q^{\prime}, \xi}$ is contained in the interval $\left(0, C(\xi) q^{\prime}\right]$.

Proof. Using polar coordinates $\left(u, q^{\prime}\right)=(r \cos \theta, r \sin \theta), \theta \in(0, \pi / 2)$, we see that inequalities (7-11) imply (7-7). This shows that for the purpose of this lemma we can replace $\Omega_{M, q^{\prime}, \xi}$ by the set of $(u, v) \in(0, Q] \times\left(0, q^{\prime}\right]$ satisfying (7-7). Therefore we can use all estimates from the first part of the proof of Lemma 9 (because they only rely on (7-7), the integrality of $q$ being used only at the end). 
Note also that $Y=Y_{M}<X=X_{M}$ and $Z^{2}=X Y-1$ yield $Y \leqslant Z$. Replacing $q$ by $u$ in the first part of the proof of Lemma 9 , so that $\tan \theta=u / q^{\prime}, \theta \in(0, \pi / 2)$, we see (compare the last line before Case 1) that $\left|\delta_{M}\right| \leqslant \Theta(\xi) / T$ for some continuous function $\Theta$. Next we look into the first estimates in Case 2 and see that there exists a function $T_{0}(\xi)$, depending continuously on $\xi$, such that, for any $M$ with $T=\|M\|^{2}>T_{0}(\xi)$, one has $0<\pi / 2-\theta<\pi / 3$ and

$$
\left|\frac{u}{q^{\prime}}-\frac{Y-\epsilon_{T}}{Z}\right| \leqslant(1+\sqrt{3})\left|\tan \delta_{M}\right| .
$$

In conjunction with the bound on $\delta_{M}$, this shows the existence of a continuous function $C_{0}(\xi)$ such that

$$
\left|u-\frac{Y-\epsilon_{T}}{Z} q^{\prime}\right| \leqslant C_{0}(\xi) q^{\prime},
$$

showing that $u \leqslant\left(1+C_{0}(\xi)\right) q^{\prime}$.

Although this will not be used in this paper, we remark that if $\gamma$ is as in (7-2), then (7-4) is satisfied by the triple $\left(q^{\prime}, q, p^{\prime}\right)$ with the pair $(Q, \xi)$ replaced by $\left(Q+\sqrt{2} Q^{c_{0}}, \xi\left(1+\sqrt{2} Q^{c_{0}-1}\right)^{2}\right)$, by the proof of (7-5). Therefore Lemma 10 shows that $q / q^{\prime} \ll \xi 1$ (with a different implicit constant than $C(\xi)$ from Lemma 10).

Next notice that, as $Q \rightarrow \infty$,

$$
\sum_{\substack{M \in \mathfrak{S} \\ \max \left\{X_{M}, Y_{M}\right\} \leqslant Q^{2 c_{0}}}} \max \left\{X_{M}, Y_{M}\right\}^{-\sigma} \ll_{\sigma} Q^{(2-2 \sigma) c_{0}}, \quad 0<\sigma<1 .
$$

This follows immediately from ${ }^{1}$

$$
\begin{gathered}
\sum_{\substack{M \in \mathfrak{S} \\
Y_{M}<X_{M} \leqslant Q^{2 c_{0}}}} X_{M}^{-\sigma} \leqslant \sum_{1 \leqslant A^{2}+B^{2} \leqslant Q^{2 c_{0}}}\left(A^{2}+B^{2}\right)^{-\sigma} \\
\leqslant \iint_{x^{2}+y^{2} \leqslant 2 Q^{2 c_{0}}}\left(x^{2}+y^{2}\right)^{-\sigma} d x d y \ll \sigma Q^{(2-2 \sigma) c_{0}} .
\end{gathered}
$$

Assume now that $Y_{M}<X_{M} \leqslant Q^{2 c_{0}}$. When $T=\|M\|^{2}>T_{0}(\xi)$ we apply Lemma 10. The definition of $\Omega$, seen after some obvious scaling as a section subset in the body $S_{M, \xi}$ defined by the conditions in (7-14) below, shows that the range of $u$ consists of a union of intervals in [0,Q] with a (universally) bounded number of components and of total Lebesgue measure $\ll \xi q^{\prime}$. This gives

$$
A(\Omega) \ll \xi \frac{Q q^{\prime}}{\sqrt{X_{M}}} \text { and } \quad \ell(\partial \Omega) \ll \xi q^{\prime}+q^{\prime} \ll \frac{Q}{\sqrt{X_{M}}} .
$$

\footnotetext{
${ }^{1}$ Here $A$ and $B$ determine uniquely the matrix $M=\left(\begin{array}{ll}A & B \\ C & D\end{array}\right)$.
} 
Taking $L=\left(q^{\prime}\right)^{5 / 6}$, we find $Q \gg X_{M}^{1 / 2}\left(q^{\prime}\right)^{1 / 6}$, and the error provided by Lemma 7 is $\mathscr{E}_{\Omega, L, q^{\prime}} \ll_{\varepsilon} Q\left(q^{\prime}\right)^{-1 / 6+\varepsilon} X_{M}^{-1 / 2}$. Note also that in this case $A \geqslant C$ and $B \geqslant D$. As a result, applying (7-12) with $\sigma=\frac{11}{12}$, the error is seen to add up to

$$
\begin{aligned}
\sum_{\substack{A^{2}+B^{2} \leqslant Q^{2 c_{0}} \\
\|M\|^{2}>T_{0}(\xi)}} \sum_{q^{\prime} \leqslant Q / \sqrt{X_{M}}} \mathscr{E}_{\Omega, q^{\prime 5 / 6}, q^{\prime}} & \ll_{\varepsilon} Q \sum_{A^{2}+B^{2} \leqslant Q^{2 c_{0}}} \frac{1}{X_{M}^{1 / 2}}\left(\frac{Q}{X_{M}^{1 / 2}}\right)^{5 / 6+\varepsilon} \\
& \ll_{\varepsilon} Q^{\left(11+c_{0}\right) / 6+\varepsilon} .
\end{aligned}
$$

Lemma 7 now provides

$$
\widetilde{\mathcal{N}}_{M}(Q, \xi)=\sum_{1 \leqslant q^{\prime} \leqslant Q / \sqrt{X_{M}}} \frac{\varphi\left(q^{\prime}\right)}{q^{\prime 2}} A\left(\Omega_{M, q^{\prime}, \xi}\right)+O_{\varepsilon}\left(Q^{\left(11+c_{0}\right) / 6+\varepsilon}\right) .
$$

The situation $\|M\|^{2} \leqslant T_{0}(\xi)$ (in this case there are $O_{\xi}(1)$ choices for $M$ ) is directly handled by Lemma 7. The same choice for $L$ provides $\mathscr{E}_{\Omega, q^{\prime 5 / 6}, q^{\prime}} \ll_{\varepsilon} Q\left(q^{\prime}\right)^{-1 / 6+\varepsilon}$. These error terms sum up to $O_{\varepsilon, \xi}\left(Q^{11 / 6+\varepsilon}\right)$ in this situation.

Next we will apply Möbius summation (see, e.g., [Boca et al. 2000, Lemma 2.3]) to the function $h_{1}\left(q^{\prime}\right)=\left(1 / q^{\prime}\right) A\left(\Omega_{M, q^{\prime}, \xi}\right)$. Note that $(1 / Q) h_{1}\left(q^{\prime}\right)$ represents the area of the cross-section of the body $S_{M, \xi}$ by the plane $x=q^{\prime} / Q$, where $S_{M, \xi}$ consists of those $(x, y, z) \in[0,1]^{3}$ such that

$$
\left|\Xi_{M}(x, y)\right| \leqslant \xi \quad \text { and } \quad x^{2} X_{M}+y^{2} Y_{M}+2 x y Z_{M} \leqslant \frac{1}{1+z^{2}} .
$$

The intersection of the projection of $S_{M, \xi}$ onto the plane $z=0$ with a vertical line $x=c$ is bounded by a quartic and an ellipse, showing that the cross-section function $c \mapsto A_{M, \xi}(c):=\operatorname{Area}\left(S_{M, \xi} \cap\{x=c\}\right)$ is continuous and piecewise $C^{1}$ on $[0,1]$ and the number of critical points of $A_{M, \xi}$ is bounded by a universal constant $C$ independently of $M$ and $\xi$. The graph on the right of Figure 6 illustrates one of the possible cases that can arise, when $A_{M, \xi}(c)$ has the most number of critical points, showing that we can take $C=3$.

In particular, the total variation of $h_{1}$ on $[0, Q]$ is bounded above by

$$
(C+1)\left(\sup _{[0, Q]} h_{1}-\inf _{[0, Q]} h_{1}\right) \ll\left\|h_{1}\right\|_{\infty} \ll \xi \frac{Q}{\sqrt{X_{M}}},
$$

and so we infer

$$
\sum_{1 \leqslant q^{\prime} \leqslant Q / \sqrt{X_{M}}} \frac{\varphi\left(q^{\prime}\right)}{q^{\prime 2}} A\left(\Omega_{M, q^{\prime}, \xi}\right)=\frac{1}{\zeta(2)} \int_{0}^{Q / \sqrt{X_{M}}} h_{1}\left(q^{\prime}\right) d q^{\prime}+O\left(\frac{Q}{\sqrt{X_{M}}} \ln Q\right) .
$$

Using also the change of variables $\left(q^{\prime}, u, v\right)=(Q x, Q y, Q x z),(x, y, z) \in[0,1]^{3}$, 

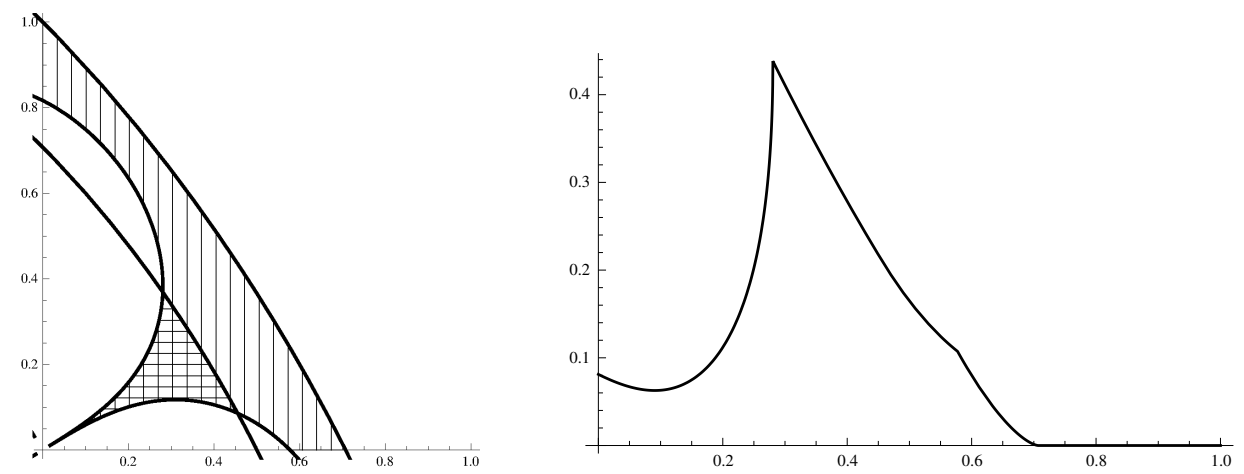

Figure 6. Left: cross-sections of $S_{M, \xi}$ for $z=0$ (vertical hatching) and $z=1$ (horizontal hatching). Right: the function $c \mapsto A_{M, \xi}(c)$, for $M=R$ and $\xi=1.5$.

(7-13), (7-5) and (7-12), we find that the contribution to $R_{Q}^{\bigcap}(\xi)$ of matrices $M$ with $Y_{M}<X_{M}$ is

$$
\begin{aligned}
& \frac{1}{\zeta(2)} \sum_{M \in \mathfrak{S}}\left(\int_{0}^{\frac{Q}{\sqrt{X_{M}}}} A\left(\Omega_{M, q^{\prime}, \xi}\right) \frac{d q^{\prime}}{q^{\prime}}+O\left(\frac{Q \ln Q}{X_{M}^{1 / 2}}\right)\right)+O_{\varepsilon, \xi}\left(Q^{\left(11+c_{0}\right) / 6+\varepsilon}\right) \\
& Y_{M}<X_{M} \leqslant Q^{2 c_{0}} \\
& =\frac{Q^{2}}{\zeta(2)} \sum_{\substack{M \in \mathfrak{S} \\
Y_{M}<X_{M} \leqslant Q^{2 c_{0}}}} \operatorname{Vol}\left(S_{M, \xi}\right)+O_{\varepsilon, \xi}\left(Q^{1+c_{0}+\varepsilon}+Q^{\left(11+c_{0}\right) / 6+\varepsilon}\right) .
\end{aligned}
$$

With $\eta=\left(\begin{array}{ll}0 & 1 \\ 1 & 0\end{array}\right)$, notice the following important symmetries:

$$
\eta M \eta=\left(\begin{array}{ll}
D & C \\
B & A
\end{array}\right) \quad \text { and } \quad \Xi_{\eta M \eta}(y, x)=-\Xi_{M}(x, y) .
$$

This shows that the reflection $(x, y, z) \mapsto(y, x, z)$ maps $S_{M, \xi}$ bijectively onto $S_{\eta M \eta, \xi}$.

The situation $X_{M}<Y_{M}$ is handled similarly using (7-16), which results in reversing the roles of $q$ and $q^{\prime}$ with Lemma 7 applied for $r=-1$.

Now we give upper bounds for $\operatorname{Vol}\left(S_{M, \xi}\right)$. Let $(x, y, z)=(r \cos t, r \sin t, z) \in S_{M, \xi}$. The proof of (7-9) and (7-10) does not use the integrality of $q^{\prime}$ and $q$, so denoting

$$
\omega_{M}=\frac{C+D}{A+B}<1 \quad \text { if } Y_{M}<X_{M} \quad \text { and } \quad \omega_{M}=\frac{A+B}{C+D}<1 \quad \text { if } X_{M}<Y_{M},
$$

we find that

$$
y \ll x \ll X_{M}^{-1 / 2} \ll T^{-1} \text { and }\left|\frac{y}{x}-\omega_{M}\right| \ll \frac{1}{T}
$$

in the former case, and

$$
x \ll y \ll Y_{M}^{-1 / 2} \ll T^{-1} \text { and }\left|\frac{x}{y}-\omega_{M}\right| \ll \frac{1}{T}
$$


in the latter case. Writing the area in polar coordinates, we find $r^{2} \ll T^{-1}$ and

$$
\begin{aligned}
\operatorname{Vol}\left(S_{M, \xi}\right) & \leqslant A\left(\left\{(x, y) \in[0,1]^{2}: \exists z \in[0,1],(x, y, z) \in S_{M, \xi}\right\}\right) \\
& \leqslant \frac{1}{2} \int_{\omega_{M}-\xi T_{M}^{-1}}^{\omega_{M}+\xi T_{M}^{-1}} 2 T_{M}^{-1} d t=\frac{2 \xi}{T_{M}^{2}}=\frac{2 \xi}{\|M\|^{4}} .
\end{aligned}
$$

The bound (7-17) and an argument similar to the proof of (7-12) yields

$$
\sum_{M \in \mathfrak{S}} \operatorname{Vol}\left(S_{M, \xi}\right)<\infty \text { and } \sum_{\substack{M \in \mathfrak{S} \\ \max \left\{X_{M}, Y_{M}\right\} \geqslant Q^{2 c_{0}}}} \operatorname{Vol}\left(S_{M, \xi}\right) \ll \xi Q^{-2 c_{0}} .
$$

From (7-15), (7-18) and $c_{0} \in\left(\frac{1}{2}, 1\right)$, we infer

$$
R_{Q}^{\Pi}(\xi)=\frac{Q^{2}}{\zeta(2)} \sum_{M \in \mathfrak{S}} \operatorname{Vol}\left(S_{M, \xi}\right)+O_{\varepsilon}\left(Q^{\left(11+c_{0}\right) / 6+\varepsilon}\right) .
$$

The volume of $S_{M, \xi}$ can be evaluated in closed form using the substitution $z=\tan t$ :

$$
\operatorname{Vol}\left(S_{M, \xi}\right)=\int_{0}^{\pi / 4} B_{M}(\xi, t) \frac{d t}{\cos ^{2} t},
$$

where $B_{M}(\xi, t)$ is the area of the region consisting of those $(r \cos \theta, r \sin \theta) \in[0,1]^{2}$ such that

$$
\frac{1}{\xi} \cdot \frac{\left|\sin \left(2 \theta-\theta_{M}\right)\right|}{U_{T}+\cos \left(2 \theta-\theta_{M}\right)} \leqslant r^{2} \leqslant \frac{1}{\sqrt{T^{2}-4}} \cdot \frac{2 \cos ^{2} t}{U_{T}+\cos \left(2 \theta-\theta_{M}\right)},
$$

with $\theta_{M} \in(0, \pi / 2)$ having $\sin \theta_{M}=2 Z_{M} / \sqrt{T^{2}-4}$ and $U_{T}=T / \sqrt{T^{2}-4}$ (for brevity we write $T=T_{M}$ ).

The following elementary fact will be useful to prove the differentiability of the volumes as functions of $\xi$.

Lemma 11. Assume that $G, H: K \rightarrow \mathbb{R}$ are continuous functions on a compact set $K \subset \mathbb{R}^{k}$, and denote $x_{+}=\max \{x, 0\}$. Then the formula

$$
V(\xi):=\int_{K}(\xi-G(v))_{+} H(v) d v, \quad \xi \in \mathbb{R},
$$

defines a $C^{1}$ map on $\mathbb{R}$, and

$$
V^{\prime}(\xi)=\int_{G<\xi} H(v) d v .
$$

Using Equation (7-20), we find that

$$
\operatorname{Vol}\left(S_{M, \xi}\right)=\frac{1}{2} \int_{0}^{\pi / 4} d t \int_{0}^{\pi / 2} d \theta \frac{\left(2 / \sqrt{T^{2}-4}-\left|\sin \left(2 \theta-\theta_{M}\right)\right| /\left(\xi \cos ^{2} t\right)\right)_{+}}{U_{T}+\cos \left(2 \theta-\theta_{M}\right)},
$$

and applying Lemma 11, we obtain: 
Corollary 12. The function $\xi \mapsto \operatorname{Vol}\left(S_{M, \xi}\right)$ is $C^{1}$.

For a smaller range for $\xi$ we have the following explicit formula:

Lemma 13. Suppose that $\xi \leqslant Z_{M}$. The volume of $S_{M, \xi}$ only depends on $\xi$ and $T=\|M\|^{2}$ :

$\operatorname{Vol}\left(S_{M, \xi}\right)$

$=\int_{0}^{\frac{\pi}{4}} \tan ^{-1}\left(\frac{\sqrt{\Delta}-\sqrt{\Delta-4 \xi^{2} \cos ^{4} t}}{2 \alpha \xi \cos ^{2} t}\right)+\frac{1}{2 \xi \cos ^{2} t} \ln \left(1-\frac{\sqrt{\Delta}-\sqrt{\Delta-4 \xi^{2} \cos ^{4} t}}{2 \alpha}\right) d t$,

where $\Delta=T^{2}-4$ and $\alpha=\frac{1}{2}\left(T+\sqrt{T^{2}-4}\right)$.

Proof. The two polar curves defined by (7-21) intersect for

$$
\left|\sin \left(2 \theta-\theta_{M}\right)\right|=\frac{2 \xi}{\sqrt{T^{2}-4}} \cos ^{2} t,
$$

that is, for $\theta_{ \pm}=\theta_{M} / 2 \pm \alpha$ with $\alpha=\alpha(\xi, t) \in(0, \pi / 4)$ such that

$$
\sin 2 \alpha=\frac{2 \xi}{\sqrt{T^{2}-4}} \cos ^{2} t
$$

Since $\sin \theta_{M}=2 Z / \sqrt{T^{2}-4}$, the assumption $\xi \leqslant Z$ ensures that $\alpha<\theta_{M}$. Thus $\theta_{ \pm} \in[0, \pi / 2)$, and the change of variables $\theta=\theta_{M} / 2+u$ yields

$$
B_{M, \xi}(t)=\frac{1}{2} \int_{-\alpha}^{\alpha}\left(\frac{2 \cos ^{2} t}{\sqrt{T^{2}-4}} \cdot \frac{1}{U_{T}+\cos (2 u)}-\frac{|\sin (2 u)|}{\xi\left(U_{T}+\cos (2 u)\right)}\right) d u .
$$

The integrand is even and both integrals can be computed exactly, yielding the formula above.

In particular, Lemma 13 yields $\operatorname{Vol}\left(S_{M, \xi}\right) \ll \xi / T^{2}$, providing an alternative proof for (7-17).

7.2. Exterior arcs. Referring to the notation of Section 5.2, we first replace the inequalities

$p^{2}+p^{\prime 2}+q^{2}+q^{\prime 2} \leqslant Q^{2} \quad$ and $\quad p_{\ell}^{2}+q_{\ell}^{2}+\left(K p_{\ell}-p_{\ell-1}\right)^{2}+\left(K q_{\ell}-q_{\ell-1}\right)^{2} \leqslant Q^{2}$

in (5-7) by simpler ones. Using $p^{\prime} q-p q^{\prime}=1$, we can replace $p$ by $p^{\prime} q / q^{\prime}$ in the former, while $p_{\ell-1}$ can be replaced by $p_{\ell} q_{\ell-1} / q_{\ell}$ in the latter. As a result, these two inequalities can be substituted in (5-7) by

$$
\begin{array}{r}
\left(1+\frac{p^{\prime 2}}{q^{\prime 2}}\right)\left(q^{2}+q^{\prime 2}\right) \leqslant Q^{2}\left(1+O\left(Q^{-1}\right)\right), \\
\left(1+\frac{p_{\ell}^{2}}{q_{\ell}^{2}}\right)\left(q_{\ell}^{2}+\left(K q_{\ell}-q_{\ell-1}\right)^{2}\right) \leqslant Q^{2}\left(1+O\left(Q^{-1}\right)\right) .
\end{array}
$$


Since $p_{\ell} / q_{\ell}=p^{\prime} / q^{\prime}+O(\ell / Q)$ and $q_{\ell}^{2}+\left(K q_{\ell}-q_{\ell-1}\right)^{2} \leqslant 2 Q^{2}$, the second inequality in (7-23) can be also written as

leading to

$$
\left(1+\frac{p^{\prime 2}}{q^{\prime 2}}\right)\left(q_{\ell}^{2}+\left(K q_{\ell}-q_{\ell-1}\right)^{2}\right) \leqslant Q^{2}\left(1+O\left(Q^{-1}\right)\right),
$$

$$
R_{Q}^{\cap \cap}(\xi)=\sum_{\substack{\ell \in[0, \xi) \\ K \in[1, \xi)}} \sum_{q^{\prime}<Q} \mathcal{N}_{Q+O\left(Q^{1 / 2}\right), q^{\prime}, K, \ell}^{\cap \cap}(\xi),
$$

where $\mathcal{N}_{Q, q^{\prime}, K, \ell}^{\cap \cap}(\xi)$ denotes the number of integer lattice points $\left(p^{\prime}, q\right)$ such that $0 \leqslant p^{\prime} \leqslant q^{\prime}, \quad 0 \leqslant q \leqslant Q, \quad p^{\prime} q \equiv 1\left(\bmod q^{\prime}\right), \quad 0<K q_{\ell}-q_{\ell-1} \leqslant Q$,

$$
\Upsilon_{\ell, K}\left(\frac{q}{Q}, \frac{q^{\prime}}{Q}\right) \leqslant \xi, \quad p^{\prime 2}+q^{\prime 2} \leqslant \frac{Q^{2} q^{\prime 2}}{\max \left\{q^{2}+q^{\prime 2}, q_{\ell}^{2}+\left(K q_{\ell}-q_{\ell-1}\right)^{2}\right\}} .
$$

Applying Lemma 7 to the set $\Omega=\Omega_{q^{\prime}, K, \ell, \xi}^{\cap \cap}$ of elements $(u, v)$ for which

$$
\begin{aligned}
u & \in[0, Q], \quad v \in\left[0, q^{\prime}\right], \quad L_{i}\left(\frac{u}{Q}, \frac{q^{\prime}}{Q}\right)>0 \text { for } i=0,1, \ldots, \ell, \\
0 & <K L_{\ell}\left(\frac{u}{Q}, \frac{q^{\prime}}{Q}\right)-L_{\ell-1}\left(\frac{u}{Q}, \frac{q^{\prime}}{Q}\right) \leqslant 1, \quad \Upsilon_{\ell, K}\left(\frac{u}{Q}, \frac{q^{\prime}}{Q}\right) \leqslant \xi, \\
v^{2}+q^{\prime 2} & \leqslant \frac{Q^{2} q^{\prime 2}}{\max \left\{u^{2}+q^{\prime 2}, Q^{2} L_{\ell}^{2}\left(\frac{u}{Q}, \frac{q^{\prime}}{Q}\right)+Q^{2}\left(K L_{\ell}\left(\frac{u}{Q}, \frac{q^{\prime}}{Q}\right)-L_{\ell-1}\left(\frac{u}{Q}, \frac{q^{\prime}}{Q}\right)\right)^{2}\right\}},
\end{aligned}
$$

with $A(\Omega) \leqslant Q q^{\prime}, \ell(\partial \Omega) \ll Q, L=\left(q^{\prime}\right)^{5 / 6}$, we find

$$
\mathcal{N}_{Q, q^{\prime}, K, \ell}^{\cap \cap}(\xi)=\frac{\varphi\left(q^{\prime}\right)}{q^{\prime}} \cdot \frac{A\left(\Omega_{q^{\prime}, K, \ell, \xi}^{\cap \cap}\right)}{q^{\prime}}+O_{\varepsilon}\left(Q\left(q^{\prime}\right)^{-1 / 6+\varepsilon}\right) .
$$

This leads in turn to

$$
R_{Q}^{\cap \cap}(\xi)=\mathcal{M}_{Q}^{\cap \cap}(\xi)+O_{\xi, \varepsilon}\left(Q^{11 / 6+\varepsilon}\right)
$$

where

$$
\mu_{Q}^{\cap \cap}(\xi)=\sum_{\substack{\ell \in[0, \xi) \\ K \in[1, \xi)}} \sum_{q^{\prime} \leqslant Q} \frac{\varphi\left(q^{\prime}\right)}{q^{\prime}} \cdot \frac{A\left(\Omega_{q^{\prime}, K, \ell, \xi}^{\cap \cap}\right)}{q^{\prime}} .
$$

For fixed integers $K \in[1, \xi), \ell \in[0, \xi)$, consider the subset $T_{K, \ell, \xi}$ of $[0,1]^{3}$ consisting of those $(x, y, z) \in[0,1]^{3}$ such that

$$
\begin{gathered}
0<L_{\ell+1}(x, y)=K L_{\ell}(x, y)-L_{\ell-1}(x, y) \leqslant 1, \quad \Upsilon_{\ell, K}(x, y) \leqslant \xi, \\
\max \left\{x^{2}+y^{2}, L_{\ell}^{2}(x, y)+L_{\ell+1}^{2}(x, y)\right\} \leqslant \frac{1}{1+z^{2}},
\end{gathered}
$$

with $L_{i}$ and $\Upsilon_{\ell, K}$ as in (5-5) and (5-6). 
Möbius summation is now applied to $h_{2}\left(q^{\prime}\right)=\left(1 / q^{\prime}\right) A\left(\Omega_{q^{\prime}, K, \ell, \xi}^{\cap \cap}\right)$. The quantity $(1 / Q) h_{2}\left(q^{\prime}\right)$ represents the area of the cross-section of the body $T_{K, \ell, \xi}$ by the plane $x=q^{\prime} / Q$. This shows that $h_{2}$ is continuous and piecewise $C^{1}$ on $[0, Q]$, and furthermore the number of critical points of $h_{2}$ is bounded uniformly in $\xi$ (and independently of $Q$ ). Hence the total variation of $h_{2}$ on $[0, Q]$ is $\ll \xi\left\|h_{2}\right\|_{\infty} \leqslant Q$. Employing also the change of variables $\left(q^{\prime}, u, v\right)=(Q x, Q y, Q x z)$, where $(x, y, z) \in[0,1]^{3}$, we find

$$
\begin{aligned}
\mu_{Q}^{\cap \cap}(\xi) & =\frac{1}{\zeta(2)} \sum_{\substack{\ell \in[0, \xi) \\
K \in[1, \xi)}}\left(\int_{0}^{Q} \frac{d q^{\prime}}{q^{\prime}} A\left(\Omega_{q^{\prime}, K, \ell, \xi}^{\cap \cap}\right)+O(Q)\right) \\
& =\frac{Q^{2}}{\zeta(2)} \sum_{\substack{\ell \in[0, \xi) \\
K \in[1, \xi)}} \operatorname{Vol}\left(T_{K, \ell, \xi}\right)+O_{\xi}(Q),
\end{aligned}
$$

and so

$$
R_{Q}^{\cap \cap}(\xi)=\frac{Q^{2}}{\zeta(2)} \sum_{\substack{\ell \in[0, \xi) \\ K \in[1, \xi)}} \operatorname{Vol}\left(T_{K, \ell, \xi}\right)+O_{\xi, \varepsilon}\left(Q^{11 / 6+\varepsilon}\right) .
$$

To show that $\xi \mapsto \operatorname{Vol}\left(T_{K, \ell, \xi}\right)$ is $C^{1}$ on $[1, \infty)$, we make the change of variables $(x, y, z)=(\cos \theta, \sin \theta, \tan t)$ to obtain

$$
\operatorname{Vol}\left(T_{K, \ell, \xi}\right)=\int_{0}^{\pi / 4} A_{K, \ell}(\xi, t) \frac{d t}{\cos ^{2} t},
$$

where $A_{K, \ell}(\xi, t)$ is the area of the region defined by the conditions in (1-3). Now notice that $K_{i}(x, y) \leqslant \xi$ when $1 \leqslant i \leqslant \ell$, as a result of (omitting the arguments of the functions)

Similarly,

$$
K_{i}=\frac{L_{i}+L_{i-2}}{L_{i-1}} \leqslant \frac{1}{L_{i-2} L_{i-1}}+\frac{1}{L_{i-1} L_{i}}<\Upsilon_{\ell, K} \leqslant \xi .
$$

$$
K_{1}=\frac{L_{-1}+L_{1}}{L_{0}} \leqslant \frac{L_{-1}}{L_{0}}+\frac{1}{L_{0} L_{1}}<\Upsilon_{\ell, K} \leqslant \xi
$$

Thus the projection of $T_{K, \ell, \xi}$ on the first two coordinates is included into the union of disjoint cylinders $\mathscr{T}_{k}:=\mathscr{T}_{k_{1}} \cap T^{-1} \mathscr{T}_{k_{2}} \cap \cdots \cap T^{-\ell+1} \mathscr{T}_{k_{\ell}}$ with $\mathscr{T}_{k}=$ $\left\{(x, y): K_{1}(x, y)=k\right\}$ and $\boldsymbol{k}=\left(k_{1}, \ldots, k_{\ell}\right) \in[1, \xi)^{\ell}$. On each set $\mathscr{T}_{\boldsymbol{k}}$ all maps $L_{1}, \ldots, L_{\ell}, L_{\ell+1}$ are linear, say $L_{i}(x, y)=A_{i} x+B_{i} y$, with integers $A_{i}, B_{i}$ depending only on $k_{1}, \ldots, k_{i}$ for $i \leqslant \ell$ and $A_{\ell+1}, B_{\ell+1}$ depending only on $\boldsymbol{k}$ and $K$. Therefore the function $F_{K, \ell}(\theta)$ is continuous on each region $\mathscr{T}_{\boldsymbol{k}}$, and applying Lemma 11 we conclude that the function $\xi \mapsto \operatorname{Vol}\left(T_{K, \ell, \xi}\right)$ is $C^{1}$ on $[1, \infty]$, being a sum of $[\xi]^{\ell}$ volumes, as functions $\rightarrow$ each of which is $C^{1}$ each of which is $C^{1}$ as a function of $\xi$. 
Remark 14. The region $T_{K, \ell, \xi}$ can be simplified further. For each integer $J \in[1, \xi)$, the map

$$
\Psi_{J}:(u, v) \mapsto\left(J L_{\ell}(u, v)-L_{\ell-1}(u, v), L_{\ell}(u, v)\right)
$$

is an area preserving injection on $\mathscr{T}$, since it is the composition of $T^{\ell}$ in (5-5) followed by the linear transformation $(u, v) \mapsto(J v-u, v)$. Note that under this map (omitting the arguments $(u, v)$ of the functions below):

$$
L_{1} \rightarrow\left[\frac{1+J L_{\ell}-L_{\ell-1}}{L_{\ell}}\right]-\left(J L_{\ell}-L_{\ell-1}\right)=L_{\ell-1}
$$

(using $L_{\ell-1}+L_{\ell}>1$ ), and by induction it follows similarly that $L_{i} \rightarrow L_{\ell-i}$ for $0 \leqslant i \leqslant \ell$. Also we have that $\Psi_{J}(u, v)=(x, y) \in[0,1]^{2}$ if and only if $x=J L_{\ell}-L_{\ell-1} \in[0,1]$ and $J=[(1+x) / y]$.

Let us decompose the region $T_{K, \ell, \xi}$ into a disjoint union of regions $T_{K, J ; \ell, \xi}$, $1 \leqslant J<\xi$, obtained by adding the condition $[(1+x) / y]=J$. By the discussion of the previous paragraph, the map $\left(\Psi_{J}, \mathrm{Id}_{z}\right)$ is a volume preserving bijection taking $U_{K, J ; \ell, \xi}$ onto $T_{K, J ; \ell, \xi}$, where $U_{K, J ; \ell, \xi}$ is the set of all $(x, y, z) \in[0,1]^{3}$ such that

$$
\begin{aligned}
& x+y>1, \quad J L_{\ell}-L_{\ell-1}>0, \quad K L_{0}-L_{1}>0, \quad \Upsilon_{\ell, K, J} \leqslant \xi, \\
& L_{0}^{2}+\left(K L_{0}-L_{1}\right)^{2} \leqslant \frac{1}{1+z^{2}}, \quad L_{\ell}^{2}+\left(J L_{\ell}-L_{\ell-1}\right)^{2} \leqslant \frac{1}{1+z^{2}} .
\end{aligned}
$$

Here $L_{i}=L_{i}(x, y)$ and

$$
\Upsilon_{\ell, K, J}(x, y)=\frac{J L_{\ell}-L_{\ell-1}}{L_{\ell}\left(L_{\ell}^{2}+\left(J L_{\ell}-L_{\ell-1}\right)^{2}\right)}+\sum_{i=1}^{\ell} \frac{1}{L_{i-1} L_{i}}+\frac{K L_{0}-L_{1}}{L_{0}\left(L_{0}^{2}+\left(K L_{0}-L_{1}\right)^{2}\right)} .
$$

For $\alpha \geqslant 1$, the transformation $\left(\Psi_{\alpha}, \mathrm{Id}_{z}\right)$ maps bijectively the part of $U_{K, J ; \ell, \xi}$ for which $\left[\left(1+L_{\ell-1}\right) / L_{\ell}\right]=\alpha$ onto the part of $U_{J, K ; \ell, \xi}$ for which $[(1+x) / y]=\alpha$. Therefore $\operatorname{Vol}\left(U_{K, J ; \ell, \xi}\right)=\operatorname{Vol}\left(U_{J, K ; \ell, \xi}\right)$ and the sum of volumes appearing in (7-28) can be written more symmetrically:

$$
\sum_{K \in[1, \xi)} \operatorname{Vol}\left(T_{K, \ell, \xi}\right)=\sum_{K, J \in[1, \xi)} \operatorname{Vol}\left(U_{K, J ; \ell, \xi}\right)
$$

As an example of using this formula, if $1<\xi \leqslant 2$ and $\ell=1$, we can only have $K=J=1$ and the inequalities $J L_{1}-L_{0}>0, K L_{0}-L_{1}>0$ cannot be both satisfied, so $U_{1,1 ; 1, \xi}$ is empty. Therefore the only contribution from the $T$ bodies in (7-28) comes from $T_{1,0, \xi}$ if $\xi \in(1,2]$.

We can now prove the main theorem on the pair correlation of the quantities $\tan \left(\theta_{\gamma} / 2\right)$. 
Theorem 2. The pair correlation measure $R_{2}^{\mathfrak{T}}$ exists on $[0, \infty)$. It is given by the $C^{1}$ function

$$
R_{2}^{\mathfrak{T}}\left(\frac{3}{8} \xi\right)=\frac{8}{3 \zeta(2)}\left(\sum_{M \in \mathfrak{S}} \operatorname{Vol}\left(S_{M, \xi}\right)+\sum_{\ell \in[0, \xi)} \sum_{K \in[1, \xi)} \operatorname{Vol}\left(T_{K, \ell, \xi}\right)\right),
$$

where the three-dimensional bodies $S_{M, \xi}$ are defined by the conditions in (7-14) and the bodies $T_{K, \ell, \xi}$ are defined by the conditions in (7-25).

Proof. By (7-19) and (7-26), with $c_{0} \in\left(\frac{1}{2}, 1\right)$ and $G(\xi)$ denoting the sum of all volumes in (7-28), we infer that

$$
\mathscr{R}_{Q}^{\Phi}(\xi)=\frac{Q^{2}}{\zeta(2)} G(\xi)+O_{\xi, \varepsilon}\left(Q^{\left(11+c_{0}\right) / 6+\varepsilon}\right) .
$$

It follows that the function $G$ is $C^{1}$ on $[0, \infty)$ as a result of $\xi \mapsto \operatorname{Vol}\left(S_{M, \xi}\right)$ being $C^{1}$ on $[0, \infty)$, and of $\xi \mapsto \operatorname{Vol}\left(T_{K, \ell, \xi}\right)$ being $C^{1}$ on $[1, \infty)$. Corollary 4 and (7-29) now yield, for $\beta \in\left(\frac{2}{3}, 1\right)$,

$\mathscr{R}_{Q}^{\Psi}(\xi)$

$=\frac{Q^{2}}{\zeta(2)}\left(G\left(\xi+O\left(Q^{2-3 \beta}\right)\right)+G\left(O\left(Q^{2-3 \beta}\right)\right)\right)+O_{\xi, \varepsilon}\left(Q^{1+\beta} \ln Q+Q^{\left(11+c_{0}\right) / 6+\varepsilon}\right)$.

Employing again the differentiability of $G$ and $G(0)=0$, and taking $\beta=\frac{3}{4}, c_{0}=\frac{1}{2}+\varepsilon$, this provides

$$
\mathscr{R}_{Q}^{\Psi}(\xi)=\frac{Q^{2}}{\zeta(2)} G(\xi)+O_{\xi, \varepsilon}\left(Q^{23 / 12+\varepsilon}\right) .
$$

Equation (7-28) now follows from (7-30) and Corollary 8.

\section{Pair correlation of $\left\{\theta_{\gamma}\right\}$}

8.1. Proof of Theorem 1. In this section we pass to the pair correlation of the angles $\left\{\theta_{\gamma}\right\}$, estimating

$$
\mathscr{R}_{Q}^{\theta}(\xi):=\#\left\{\left(\gamma, \gamma^{\prime}\right) \in \widetilde{\mathfrak{R}}_{Q}^{2}: 0 \leqslant Q^{2}\left(\theta_{\gamma^{\prime}}-\theta_{\gamma}\right) \leqslant \xi\right\} .
$$

Define the pair correlation kernel $F(\xi, t)$ as follows:

$$
F(\xi, t)=\sum_{M \in \mathfrak{S}} B_{M}(\xi, t)+\sum_{\substack{\ell \in[0, \xi) \\ K \in[1, \xi)}} A_{K, \ell}(\xi, t),
$$

where $B_{M}(\xi, t)$ and $A_{K, \ell}(\xi, t)$ are the areas from (7-20) and (7-27), respectively, so that by (7-30) we have

$$
\mathscr{R}_{Q}^{\Psi}(\xi)=\frac{Q^{2}}{\zeta(2)} \int_{0}^{\pi / 4} F(\xi, t) \frac{d t}{\cos ^{2} t}+O_{\xi, \varepsilon}\left(Q^{\left(11+c_{0}\right) / 6+\varepsilon}\right) .
$$


Proposition 15. $\mathscr{R}_{Q}^{\theta}(\xi)=\frac{Q^{2}}{\zeta(2)} \int_{0}^{\pi / 4} F\left(\frac{\xi}{2 \cos ^{2} t}, t\right) \frac{d t}{\cos ^{2} t}+O_{\xi, \varepsilon}\left(Q^{47 / 24+\varepsilon}\right)$.

Before giving the proof, note that Theorem 1 follows from the proposition as $Q \rightarrow \infty$, taking into account the different normalization in the definitions of $\mathscr{R}_{Q}^{\theta}(\xi)$ and $R_{Q}^{\mathfrak{A}}(\xi)$, and defining, in view of Proposition 15 and (8-1),

$$
B_{M}(\xi):=\int_{0}^{\frac{\pi}{4}} B_{M}\left(\frac{\xi}{2 \cos ^{2} t}, t\right) \frac{d t}{\cos ^{2} t}, \quad A_{K, \ell}(\xi):=\int_{0}^{\frac{\pi}{4}} A_{K, \ell}\left(\frac{\xi}{2 \cos ^{2} t}, t\right) \frac{d t}{\cos ^{2} t} .
$$

From the definitions of $B_{M}(\xi, t), A_{K, \ell}(\xi, t)$ in the equations following (7-20), (7-27), it is clear that

$$
B_{M}\left(\frac{\xi}{2 \cos ^{2} t}, t\right)=B_{M}\left(\frac{\xi}{2}, 0\right) \cos ^{2} t, \quad A_{K, 0}\left(\frac{\xi}{2 \cos ^{2} t}, t\right)=A_{K, 0}\left(\frac{\xi}{2}, 0\right) \cos ^{2} t,
$$

hence one has

$$
B_{M}(\xi)=\frac{\pi}{4} B_{M}\left(\frac{\xi}{2}, 0\right), \quad A_{K, 0}(\xi)=\frac{\pi}{4} A_{K, 0}\left(\frac{\xi}{2}, 0\right),
$$

which together with (7-22) yields the formula for $B_{M}(\xi)$ given in Theorem 1. Note that the range of summation in Theorem 1 restricts to $K<\xi / 2, \ell<\xi / 2$, compared with the range in (8-1). Indeed, from the description of $A_{K, \ell}\left(\xi / 2 \cos ^{2} t, t\right)$ following (7-27), we see that $\ell<\Upsilon_{\ell, K} \leqslant \xi / 2$, while for $K$ we have

$$
K<\frac{1}{L_{\ell-1} L_{\ell}}+\frac{K L_{\ell}-K_{\ell-1}}{L_{\ell}}<\Upsilon_{\ell, K} \leqslant \frac{\xi}{2},
$$

and similarly for $\ell=0$.

Proof of Proposition 15. Consider $I=[\alpha, \beta)$ with $N=\left[Q^{d}\right],|I|=N^{-1} \sim Q^{-d}$, $I^{+}=\left[\alpha-Q^{-d^{\prime}}, \beta+Q^{-d^{\prime}}\right]$, and $I^{-}=\left[\alpha+Q^{-d^{\prime}}, \beta-Q^{-d^{\prime}}\right]$, where

$$
0<d=\frac{1}{24}<d^{\prime}=\frac{1}{12}<1 .
$$

Partition the interval $[0,1)$ into the union of $N$ intervals $I_{j}=\left[\alpha_{j}, \alpha_{j+1}\right)$, with $\left|I_{j}\right|=N^{-1}$ as above. Associate the intervals $I_{j}^{ \pm}$to $I_{j}$ as described above. Set

$$
\begin{aligned}
& \mathfrak{R}_{Q}^{\sharp}:=\left\{\left(\gamma, \gamma^{\prime}\right) \in \widetilde{\mathfrak{R}}_{Q}^{2}: \gamma \neq \gamma^{\prime}\right\}, \\
& \mathscr{R}_{I, Q}^{\theta}(\xi):=\#\left\{\left(\gamma, \gamma^{\prime}\right) \in \mathfrak{R}_{Q}^{\sharp}: 0 \leqslant Q^{2}\left(\theta_{\gamma^{\prime}}-\theta_{\gamma}\right) \leqslant \xi, \Psi(\gamma), \Psi\left(\gamma^{\prime}\right) \in I\right\}, \\
& \mathscr{R}_{I, Q}^{\theta, \natural}(\xi):=\#\left\{\left(\gamma, \gamma^{\prime}\right) \in \mathfrak{R}_{Q}^{\sharp}: 0 \leqslant Q^{2}\left(\theta_{\gamma^{\prime}}-\theta_{\gamma}\right) \leqslant \xi, \Psi(\gamma) \in I\right\} \quad\left(\geqslant \mathscr{R}_{I, Q}^{\theta}(\xi)\right), \\
& \mathscr{R}_{I, Q}^{\Psi}(\xi):=\#\left\{\left(\gamma, \gamma^{\prime}\right) \in \mathfrak{R}_{Q}^{\sharp}: 0 \leqslant Q^{2}\left(\Psi\left(\gamma^{\prime}\right)-\Psi(\gamma)\right) \leqslant \xi, \Psi(\gamma), \Psi\left(\gamma^{\prime}\right) \in I\right\}, \\
& \mathscr{R}_{I, Q}^{\Psi, b}(\xi):=\#\left\{\left(\gamma, \gamma^{\prime}\right) \in \mathfrak{R}_{Q}^{\sharp}: 0 \leqslant Q^{2}\left(\Psi\left(\gamma^{\prime}\right)-\Psi(\gamma)\right) \leqslant \xi, \gamma_{-}, \gamma_{+} \in I\right\}, \\
& \mathscr{R}_{I, Q}^{\Phi, b}(\xi):=\#\left\{\left(\gamma, \gamma^{\prime}\right) \in \mathfrak{R}_{Q}^{\sharp}: 0 \leqslant Q^{2}\left(\Phi\left(\gamma^{\prime}\right)-\Phi(\gamma)\right) \leqslant \xi, \gamma_{-}, \gamma_{+} \in I\right\} .
\end{aligned}
$$


Expressing $\theta_{\gamma^{\prime}}-\theta_{\gamma}$ and $\Psi\left(\gamma^{\prime}\right)-\Psi(\gamma)$ via the mean value theorem, we find

$$
\mathscr{R}_{I, Q}^{\Psi}\left(\frac{1}{2}\left(1+\alpha^{2}\right) \xi\right) \leqslant \mathscr{R}_{I, Q}^{\theta}(\xi) \leqslant \mathscr{R}_{I, Q}^{\Psi}\left(\frac{1}{2}\left(1+\beta^{2}\right) \xi\right) .
$$

Lemma 16. The following estimates hold:

(i) $\sum_{j=1}^{N} \mathscr{R}_{I_{j}, Q}^{\theta}(\xi) \leqslant \mathscr{R}_{Q}^{\theta}(\xi)=\sum_{j=1}^{N} \mathscr{R}_{I_{j}, Q}^{\theta, \natural}(\xi) \leqslant \sum_{j=1}^{N} \mathscr{R}_{I_{j}^{+}, Q}^{\theta}(\xi)+O\left(Q^{15 / 8} \ln ^{2} Q\right)$.

(ii) $\mathscr{R}_{I, Q}^{\Psi}(\xi)=\mathscr{R}_{I, Q}^{\Psi, b}(\xi)+O\left(Q^{1+d^{\prime}} \ln ^{2} Q\right)$.

Proof. The first inequality in (i) is trivial. For the second one, note first that the total number of pairs $\left(\gamma, \gamma^{\prime}\right)$ with $0 \leqslant \theta_{\gamma^{\prime}}-\theta_{\gamma} \leqslant \xi Q^{-2}$ and $q q^{\prime} \leqslant Q^{d^{\prime}}$, with $\gamma_{-}=p / q$ and $\gamma_{+}=p^{\prime} / q^{\prime}$, is $\ll_{\xi} Q^{d}\left(Q^{d^{\prime}} \ln Q\right)(Q \ln Q)$. For $\gamma$ with $q q^{\prime}>Q^{-d^{\prime}}$, use $\Psi\left(\gamma^{\prime}\right)-\beta \leqslant \Psi\left(\gamma^{\prime}\right)-\Psi(\gamma) \leqslant 1 / q q^{\prime} \leqslant Q^{-d^{\prime}}$, so $\Psi\left(\gamma^{\prime}\right) \in I_{j}^{+}$. The proof of (ii) is analogous.

Lemma 16 and (8-3) yield

$$
\sum_{j=1}^{N} \mathscr{R}_{I_{j}, Q}^{\Psi}\left(\frac{1}{2}\left(1+\alpha_{j}^{2}\right) \xi\right) \leqslant \mathscr{R}_{Q}^{\theta}(\xi) \leqslant \sum_{j=1}^{N} \mathscr{R}_{I_{j}^{+}, Q}^{\Psi}\left(\frac{1}{2}\left(1+\alpha_{j+1}^{2}\right) \xi\right)+O_{\varepsilon}\left(Q^{15 / 8+\varepsilon}\right) .
$$

To estimate $\mathscr{R}_{I, Q}^{\Phi}(\xi)$ we repeat the previous arguments for a short interval $I$ as above. Adding everywhere the condition $\gamma_{-}, \gamma_{+} \in I$, we modify $\mathscr{R}_{Q}^{\mathrm{n}}$ by $\mathscr{R}_{I, Q}^{\mathrm{n}}$ and $R_{Q}^{\cap}$ by $R_{I, Q}^{\bigcap}$ in Lemma $5, \mathscr{R}_{Q}^{\cap \cap}$ by $\mathscr{R}_{I, Q}^{\cap \cap}$ and $R_{Q}^{\cap \cap}$ by $R_{I, Q}^{\cap \cap}$ in Lemma 6 . The additional condition $p / q, p^{\prime} / q^{\prime} \in I$ is inserted in (7-2). The condition $0 \leqslant p^{\prime} \leqslant q^{\prime}$ is replaced by $q^{\prime} \alpha \leqslant p^{\prime}<q^{\prime} \beta$ in (7-4) and (7-24), and $0 \leqslant p \leqslant q$ is replaced by $q \alpha \leqslant p<q \beta$ in (7-4). The condition $v \in\left[0, q^{\prime}\right]$ is replaced by $v \in\left[q^{\prime} \alpha, q^{\prime} \beta\right)$ in the definition of $\Omega_{M, q^{\prime}, \xi}$, and $\Omega_{q^{\prime}, \ell, K, \xi}^{\cap}$. The bodies $S_{M, \xi}$ and $T_{K, \ell, \xi}$ are substituted, respectively, by $S_{I, M, \xi}$ and $T_{I, K, \ell, \xi}$ after replacing the condition $z \in[0,1]$ in their definitions by $z \in[\alpha, \beta)$. The analogs of (7-20) and (7-27) hold:

$$
\operatorname{Vol}\left(S_{I, M, \xi}\right)=\int_{I} B_{M}(\xi, t) \frac{d t}{\cos ^{2} t}, \quad \operatorname{Vol}\left(T_{I, K, l, \xi}\right)=\int_{I} A_{K, \ell}(\xi, t) \frac{d t}{\cos ^{2} t} .
$$

The approach from Section 7 under the changes specified in the previous paragraph leads to

$$
R_{I, Q}^{\Phi, b}(\xi)=R_{I, Q}^{\bigcap}(\xi)+R_{I, Q}^{\cap \cap}(\xi)=\frac{Q^{2}}{\zeta(2)} \int_{\tan ^{-1} I} F(\xi, t) \frac{d t}{\cos ^{2} t}+O_{\xi, \varepsilon}\left(Q^{23 / 12+\varepsilon}\right),
$$

with the pair correlation kernel $F(\xi, t)$ defined by (8-1). We also have

$$
R_{I^{+}, Q}^{\Phi, b}(\xi)=R_{I, Q}^{\Phi, b}(\xi)+O_{\xi, \varepsilon}\left(Q^{23 / 12+\varepsilon}+Q^{2-d^{\prime}}\right) .
$$


1032Florin P. Boca, Vicentiu Pasol, Alexandru A. Popa and Alexandru Zaharescu

The analogs of Lemmas 5 and 6 yield, upon (8-5) and (8-6),

$\mathscr{R}_{I, Q}^{\Phi, b}(\xi)=\frac{Q^{2}}{\zeta(2)} \int_{\tan ^{-1} I} F\left(\xi+O\left(Q^{-1 / 3}\right), t\right) \frac{d t}{\cos ^{2} t}+O_{\xi, \varepsilon}\left(Q^{23 / 12+\varepsilon}\right)=\mathscr{R}_{I^{+}, Q}^{\Phi, b}(\xi)$.

The analog of Corollary 4 and (8-7) yield

$$
\begin{aligned}
\mathscr{R}_{I, Q}^{\Psi, b}(\xi)=\mathscr{R}_{I, Q}^{\Phi, b}\left(\xi+O\left(Q^{-1 / 4}\right)\right)+\mathscr{R}_{I, Q}^{\Phi, b}\left(O\left(Q^{-1 / 4}\right)\right)+O_{\varepsilon}\left(Q^{7 / 4+\varepsilon}\right) & \\
\left.=\frac{Q^{2}}{\zeta(2)} \int_{\tan ^{-1} I}\left(F\left(\xi+O\left(Q^{-1 / 4}\right), t\right)+F\left(Q^{-1 / 4}, t\right)\right)\right) \frac{d t}{\cos ^{2} t} & +O_{\xi, \varepsilon}\left(Q^{23 / 12+\varepsilon}\right) \\
& =\mathscr{R}_{I^{+}, Q^{\Psi, b}}(\xi)
\end{aligned}
$$

As shown in Section 7, the function $F$ is $C^{1}$ in $\xi$, thus (8-8) gives actually ${ }^{2}$

$$
\mathscr{R}_{I, Q}^{\Psi, b}(\xi)=\frac{Q^{2}}{\zeta(2)} \int_{\tan ^{-1} I} F(\xi, t) \frac{d t}{\cos ^{2} t}+O_{\xi, \varepsilon}\left(Q^{23 / 12+\varepsilon}\right)=\mathscr{R}_{I^{+}, Q}^{\Psi, b}(\xi)
$$

Lemma 16(i), (8-9), and the fact that $F \in C^{1}[0, \infty)$ yield

$$
\mathscr{R}_{I, Q}^{\Psi}(\xi)=\frac{Q^{2}}{\zeta(2)} \int_{\tan ^{-1} I} F(\xi, t) \frac{d t}{\cos ^{2} t}+O_{\xi, \varepsilon}\left(Q^{23 / 12+\varepsilon}+Q^{2-d^{\prime}}\right)=\mathscr{R}_{I^{+}, Q}^{\Psi}(\xi) .
$$

Let also $\omega_{j}=\tan ^{-1} \alpha_{j}$. From (8-10) and (8-3) we further infer

$$
\begin{array}{rl}
\frac{Q^{2}}{\zeta(2)} \int_{\omega_{j}}^{\omega_{j+1}} & F\left(\frac{1}{2}\left(1+\alpha_{j}^{2}\right) \xi, t\right) \frac{d t}{\cos ^{2} t}+O_{\xi, \varepsilon}\left(Q^{23 / 12+\varepsilon}+Q^{2-d^{\prime}}\right) \\
& \leqslant \mathscr{R}_{I_{j}, Q}^{\theta}(\xi) \leqslant \mathscr{R}_{I_{j}^{+}, Q}^{\theta}(\xi) \\
& \leqslant \frac{Q^{2}}{\zeta(2)} \int_{\omega_{j}}^{\omega_{j+1}} F\left(\frac{1}{2}\left(1+\alpha_{j+1}^{2}\right) \xi, t\right) \frac{d t}{\cos ^{2} t}+O_{\xi, \varepsilon}\left(Q^{23 / 12+\varepsilon}+Q^{2-d^{\prime}}\right) .
\end{array}
$$

Employing also

$\int_{\omega_{j}}^{\omega_{j+1}} F\left(\frac{1}{2}\left(1+\alpha_{j}^{2}\right) \xi, t\right) \frac{d t}{\cos ^{2} t}=\int_{\omega_{j}}^{\omega_{j+1}}\left(F\left(\frac{1}{2}\left(1+\tan ^{2} t\right) \xi, t\right)+O\left(\omega_{j+1}-\omega_{j}\right)\right) \frac{d t}{\cos ^{2} t}$

and $\left(\omega_{j+1}-\omega_{j}\right)^{2} \leqslant Q^{-2 d}$, we find that

$\mathscr{R}_{I_{j}, Q}^{\theta}(\xi)=\frac{Q^{2}}{\zeta(2)} \int_{\omega_{j}}^{\omega_{j+1}} F\left(\frac{1}{2}\left(1+\tan ^{2} t\right) \xi, t\right) \frac{d t}{\cos ^{2} t}+O_{\xi, \varepsilon}\left(Q^{23 / 12+\varepsilon}\right)=\mathscr{R}_{I_{j}^{+}, Q}^{\theta}(\xi)$.

This, together with Lemma 16(i), yields the equality from Proposition 15.

\footnotetext{
${ }^{2}$ The argument from Section 7 applies before integrating with respect to $t$ on $[0, \pi / 4]$, showing that $F$ is $C^{1}$.
} 
8.2. Explicit formula for $\boldsymbol{g}_{2}^{\mathfrak{A}}$. Next we compute the derivatives $B_{M}^{\prime}(\xi)$, thus proving Corollary 1. We also obtain the explicit formula (8-11) for $g_{2}^{\mathfrak{A}}$ on a larger range than in Corollary 1, after computing the derivative $A_{K, 0}^{\prime}(\xi)$.

Lemma 17. For $M \in \mathfrak{S}$, let $T=T_{M}, Z=Z_{M}$ as in (3-1). The derivative $B_{M}^{\prime}(\xi)$ is given by

$$
\begin{cases}\frac{\pi}{4 \xi^{2}} \ln \left(\frac{T+\sqrt{T^{2}-4}}{T+\sqrt{T^{2}-4-\xi^{2}}}\right) & \text { if } \xi \leqslant 2 Z, \\ \frac{\pi}{8 \xi^{2}} \ln \left(\frac{\left(T+\sqrt{T^{2}-4}\right)^{2}\left(T-\sqrt{T^{2}-4-\xi^{2}}\right)}{\left(4+4 Z^{2}\right)\left(T+\sqrt{T^{2}-4-\xi^{2}}\right)}\right) & \text { if } 2 Z \leqslant \xi \leqslant \sqrt{T^{2}-4}, \\ \frac{\pi}{8 \xi^{2}} \ln \left(\frac{\left(T+\sqrt{T^{2}-4}\right)^{2}}{4+4 Z^{2}}\right) & \text { if } \xi \geqslant \sqrt{T^{2}-4} .\end{cases}
$$

Proof. Using (8-2), we proceed as in the proof of Lemma 13:

$$
B_{M}(\xi)=\frac{\pi}{4 \xi} \int_{0}^{\pi / 2}\left(\frac{\xi}{\sqrt{T^{2}-4}} \cdot \frac{1}{U_{T}+\cos \left(2 \theta-\theta_{M}\right)}-\frac{\left|\sin \left(2 \theta-\theta_{M}\right)\right|}{U_{T}+\cos \left(2 \theta-\theta_{M}\right)}\right)_{+} d \theta,
$$

where $U_{T}=T / \sqrt{T^{2}-4}$, and $\theta_{M} \in(0, \pi / 2)$ has $\sin \theta_{M}=2 Z / \sqrt{T^{2}-4}$. Applying Lemma 11, we obtain

$$
B_{M}^{\prime}(\xi)=\frac{\pi}{4 \xi^{2}} \int_{I} \frac{\left|\sin \left(2 \theta-\theta_{M}\right)\right|}{U_{T}+\cos \left(2 \theta-\theta_{M}\right)} d \theta,
$$

with $I=\left\{\theta \in(0, \pi / 2):\left|\sin \left(2 \theta-\theta_{M}\right)\right|<\xi / \sqrt{T^{2}-4}\right\}$. Clearly $I=(0, \pi / 2)$ when $\xi>\sqrt{T^{2}-4}$, and if $\xi \leqslant \sqrt{T^{2}-4}$, let $\alpha=\alpha(\xi) \in(0, \pi / 4)$ be such that $\sin 2 \alpha=\xi / \sqrt{T^{2}-4}$. Then

$$
\begin{aligned}
\xi \leqslant 2 Z & \Longleftrightarrow \alpha \leqslant \theta_{M} / 2 \Longleftrightarrow I=\left[\theta_{M} / 2-\alpha, \theta_{M} / 2+\alpha\right], \\
2 Z \leqslant \xi \leqslant \sqrt{T^{2}-4} & \Longleftrightarrow \alpha \in\left[\theta_{M} / 2, \pi / 4\right] \\
& \Longleftrightarrow I=\left[0, \theta_{M} / 2+\alpha\right] \cup\left[\pi / 2+\theta_{M} / 2-\alpha, \pi / 2\right],
\end{aligned}
$$

and the integral is easy to compute. For $M=\left(\begin{array}{ll}1 & 1 \\ 0 & 1\end{array}\right)$ and $\xi=3$, the region with area $B_{M}(\xi / 2,0)$ is the one hatched vertically in Figure 6.

A similar computation using (8-2) shows that $A_{K, 0}^{\prime}(\xi)$ is given by

$$
\frac{\pi}{4 \xi^{2}} \cdot \begin{cases}0 & \text { if } \xi \leqslant 2 K, \\ \ln \left(1+K^{2}\right)+\ln \left(\frac{\left(1+x_{1}^{2}\right)\left(1+\left(x_{2}-K\right)^{2}\right)}{\left(1+x_{2}^{2}\right)\left(1+\left(x_{1}-K\right)^{2}\right)}\right) & \text { if } \xi \in\left[2 K, K \sqrt{K^{2}+4}\right], \\ \ln \left(1+K^{2}\right) & \text { if } \xi \geqslant K \sqrt{K^{2}+4},\end{cases}
$$

where $x_{2}>x_{1}$ are the roots of

$$
x^{2}(\xi+2 K)-2 x K(\xi+K)+\xi\left(K^{2}+1\right)-2 K=0 .
$$


By the last paragraph in Remark 14, the body $T_{1,1, \xi}$ is empty, so $A_{1,1}(\xi)=0$, and we have an explicit formula on a larger range than in the introduction:

$$
g_{2}^{\mathfrak{A}}\left(\frac{3}{4 \pi} \xi\right)=\frac{32 \pi}{9 \zeta(2)}\left(\sum_{M \in \mathfrak{S}} B_{M}^{\prime}(\xi)+A_{1,0}^{\prime}(\xi)\right), \quad 0<\xi \leqslant 4 .
$$

We can now explain the presence of the spikes in the graph of $g_{2}^{\mathfrak{A}}$ in Figure 1. The function $B_{M}^{\prime}(\xi)$ is not differentiable at $\xi=2 F$ and $\sqrt{T^{2}-4}$, while the function $A_{K, 0}^{\prime}(\xi)$ is not differentiable at $\xi=2 K$ and $\sqrt{\left(K^{2}+2\right)^{2}-4}$. At the point $\xi=\sqrt{5}$, two of the functions $B_{M}^{\prime}(\xi)$, as well as $A_{1,0}^{\prime}(\xi)$, have infinite slopes on the left, which gives the spike on the graph of $g_{2}^{\mathfrak{A}}(x)$ at $x=(3 / 4 \pi) \sqrt{5}$.

\section{Acknowledgments}

We thank the referees for their constructive comments, which led to improvements and clarifications in the presentation. Boca and Popa were supported in part by the CNCS - UEFISCDI grant PN-II-RU-TE-2011-3-0259. Paşol was supported in part by grant CNCSIS RU-TE-2011-3-0053. Popa was supported in part by European Community's Marie Curie grant PIRG05-GA-2009-248569. Zaharescu was supported in part by NSF grant DMS-0901621.

\section{References}

[Boca 2007] F. P. Boca, "Distribution of angles between geodesic rays associated with hyperbolic lattice points”, Q. J. Math. 58:3 (2007), 281-295. MR 2008j:11142 Zbl 1197.11134

[Boca and Zaharescu 2005] F. P. Boca and A. Zaharescu, "The correlations of Farey fractions", J. London Math. Soc. (2) 72:1 (2005), 25-39. MR 2006h:11086 Zbl 1089.11037

[Boca et al. 2000] F. P. Boca, C. Cobeli, and A. Zaharescu, "Distribution of lattice points visible from the origin", Comm. Math. Phys. 213:2 (2000), 433-470. MR 2001j:11094 Zbl 0989.11049

[Boca et al. 2001] F. P. Boca, C. Cobeli, and A. Zaharescu, "A conjecture of R. R. Hall on Farey points”, J. Reine Angew. Math. 535 (2001), 207-236. MR 2002f:11127 Zbl 1006.11053

[Boca et al. 2013] F. P. Boca, A. A. Popa, and A. Zaharescu, "Pair correlation of hyperbolic lattice angles", preprint, 2013. arXiv 1302.5067

[Bosma et al. 1997] W. Bosma, J. Cannon, and C. Playoust, “The Magma algebra system, I: The user language”, J. Symbolic Comput. 24:3-4 (1997), 235-265. MR 1484478 Zbl 0898.68039

[Chamizo 2006] F. Chamizo, "Non-Euclidean visibility problems", Proc. Indian Acad. Sci. Math. Sci. 116:2 (2006), 147-160. MR 2006m:52012 Zbl 1126.11033

[Estermann 1961] T. Estermann, “On Kloosterman's sum”, Mathematika 8 (1961), 83-86. MR 23 \#A3716 Zbl 0114.26302

[Fricke and Klein 1892] R. Fricke and F. Klein, Vorlesungen über die Theorie der Elliptischen Modulfunktionen, II, Teubner, Leipzig, 1892.

[Good 1983] A. Good, "On various means involving the Fourier coefficients of cusp forms", Math. Z. 183:1 (1983), 95-129. MR 84g:10052 Zbl 0497.10015

[Gorodnik and Nevo 2012] A. Gorodnik and A. Nevo, "Counting lattice points", J. Reine Angew. Math. 663 (2012), 127-176. MR 2889708 Zbl 1248.37011 
[Hall and Tenenbaum 1984] R. R. Hall and G. Tenenbaum, "On consecutive Farey arcs", Acta Arith. 44:4 (1984), 397-405. MR 86e:11087 Zbl 0553.10011

[Hooley 1957] C. Hooley, "An asymptotic formula in the theory of numbers", Proc. London Math. Soc. (3) 7 (1957), 396-413. MR 19,839c Zbl 0079.27301

[Iwaniec 2002] H. Iwaniec, Spectral methods of automorphic forms, 2nd ed., Graduate Studies in Mathematics 53, Amer. Math. Soc., Providence, RI, 2002. MR 2003k:11085 Zbl 1006.11024

[Kelmer and Kontorovich 2013] D. Kelmer and A. Kontorovich, "On the pair correlation density for hyperbolic angles", preprint, 2013. arXiv 1308.0754

[Narkiewicz 1983] W. Narkiewicz, Number theory, World Scientific, Singapore, 1983. MR 85j:11002 Zbl 0528.10001

[Nicholls 1983] P. Nicholls, "A lattice point problem in hyperbolic space", Michigan Math. J. 30:3 (1983), 273-287. MR 85f:11071 Zbl 0537.30033

[Nicholls 1989] P. J. Nicholls, The ergodic theory of discrete groups, London Mathematical Society Lecture Note Series 143, Cambridge University Press, 1989. MR 91i:58104 Zbl 0674.58001

[Pollicott and Sharp 2006] M. Pollicott and R. Sharp, "Correlations for pairs of closed geodesics", Invent. Math. 163:1 (2006), 1-24. MR 2007a:37036 Zbl 1081.37016

[Risager and Rudnick 2009] M. S. Risager and Z. Rudnick, "On the statistics of the minimal solution of a linear Diophantine equation and uniform distribution of the real part of orbits in hyperbolic spaces", pp. 187-194 in Spectral analysis in geometry and number theory (Nagoya, 2007), edited by M. Kotani et al., Contemp. Math. 484, Amer. Math. Soc., Providence, RI, 2009. MR 2010b:11076 Zbl 1189.11036

[Risager and Truelsen 2010] M. S. Risager and J. L. Truelsen, "Distribution of angles in hyperbolic lattices", Q. J. Math. 61:1 (2010), 117-133. MR 2011i:11102 Zbl 1194.11081

[Sarnak 2007] P. Sarnak, "Reciprocal geodesics", pp. 217-237 in Analytic number theory (Göttingen, 2005), edited by W. Duke and Y. Tschinkel, Clay Math. Proc. 7, Amer. Math. Soc., Providence, RI, 2007. MR 2009a:11184 Zbl 1198.11039

[Stein et al. 2012] W. A. Stein et al., Sage Mathematics Software (Version 5.0.1), 2012, Available at http://www.sagemath.org.

[Weil 1948] A. Weil, "On some exponential sums", Proc. Nat. Acad. Sci. U. S. A. 34 (1948), 204-207. MR 10,234e Zbl 0032.26102

[Yao and Knuth 1975] A. C. Yao and D. E. Knuth, "Analysis of the subtractive algorithm for greatest common divisors", Proc. Nat. Acad. Sci. U.S.A. 72:12 (1975), 4720-4722. MR 54 \#5102 Zbl 0315.10005

Communicated by Brian Conrad

Received 2013-09-03 Revised 2013-10-21 Accepted 2013-11-22

fboca@illinois.edu

Department of Mathematics, University of Illinois, 1409 W. Green Street, Urbana, IL 61801, United States

vicentiu.pasol@imar.ro

Simion Stoilow Institute of Mathematics of the Romanian Academy, P.O. Box 1-764, 014700 Bucharest, Romania

alexandru.popa@imar.ro

Simion Stoilow Institute of Mathematics of the Romanian Academy, P.O. Box 1-764, 014700 Bucharest, Romania

zaharesc@illinois.edu

Department of Mathematics, University of Illinois, 1409 W. Green Street, Urbana, IL 61801, United States 


\section{Algebra \& Number Theory}

msp.org/ant

\section{EDITORS}

MANAGING EDITOR

Bjorn Poonen

Massachusetts Institute of Technology

Cambridge, USA

\author{
EDITORIAL BOARD CHAIR \\ David Eisenbud \\ University of California \\ Berkeley, USA
}

BOARD OF EDITORS

Georgia Benkart

Dave Benson

Richard E. Borcherds

John H. Coates

J-L. Colliot-Thélène

Brian D. Conrad

Hélène Esnault

Hubert Flenner

Edward Frenkel

Andrew Granville

Joseph Gubeladze

Roger Heath-Brown

Craig Huneke

Yujiro Kawamata

János Kollár

Yuri Manin

Barry Mazur

Philippe Michel

Susan Montgomery
University of Wisconsin, Madison, USA

University of Aberdeen, Scotland

University of California, Berkeley, USA

University of Cambridge, UK

CNRS, Université Paris-Sud, France

University of Michigan, USA

Freie Universität Berlin, Germany

Ruhr-Universität, Germany

University of California, Berkeley, USA

Université de Montréal, Canada

San Francisco State University, USA

Oxford University, UK

University of Virginia, USA

University of Tokyo, Japan

Princeton University, USA

Northwestern University, USA

Harvard University, USA

École Polytechnique Fédérale de Lausanne

University of Southern California, USA
Shigefumi Mori

Raman Parimala

Jonathan Pila

Anand Pillay

Victor Reiner

Peter Sarnak

Joseph H. Silverman

Michael Singer

Vasudevan Srinivas

J. Toby Stafford

Bernd Sturmfels

Richard Taylor

Ravi Vakil

Michel van den Bergh

Marie-France Vignéras

Kei-Ichi Watanabe

Efim Zelmanov

Shou-Wu Zhang
RIMS, Kyoto University, Japan

Emory University, USA

University of Oxford, UK

University of Notre Dame, USA

University of Minnesota, USA

Princeton University, USA

Brown University, USA

North Carolina State University, USA

Tata Inst. of Fund. Research, India

University of Michigan, USA

University of California, Berkeley, USA

Harvard University, USA

Stanford University, USA

Hasselt University, Belgium

Université Paris VII, France

Nihon University, Japan

University of California, San Diego, USA

Princeton University, USA

\section{PRODUCTION}

production@msp.org

Silvio Levy, Scientific Editor

See inside back cover or msp.org/ant for submission instructions.

The subscription price for 2014 is US $\$ 225 /$ year for the electronic version, and $\$ 400 /$ year ( $\$ 55$, if shipping outside the US) for print and electronic. Subscriptions, requests for back issues and changes of subscribers address should be sent to MSP.

Algebra \& Number Theory (ISSN 1944-7833 electronic, 1937-0652 printed) at Mathematical Sciences Publishers, 798 Evans Hall \#3840, c/o University of California, Berkeley, CA 94720-3840 is published continuously online. Periodical rate postage paid at Berkeley, CA 94704, and additional mailing offices.

ANT peer review and production are managed by EditFLOw ${ }^{\circledR}$ from Mathematical Sciences Publishers.

\section{PUBLISHED BY}

mathematical sciences publishers

nonprofit scientific publishing

http://msp.org/

(C) 2014 Mathematical Sciences Publishers 


\section{Algebra \& Number Theory}

Volume $8 \quad$ No. $4 \quad 2014$

The derived moduli space of stable sheaves

Kai Behrend, Ionut Ciocan-Fontanine, Junho Hwang and Michael Rose

Averages of the number of points on elliptic curves

Greg Martin, Paul Pollack and Ethan Smith

Noncrossed product bounds over Henselian fields

Timo Hanke, DanNy NeFtin and JaCk SONN

Yangians and quantizations of slices in the affine Grassmannian

Joel Kamnitzer, Ben Webster, Alex Weekes and Oded Yacobi

Equidistribution of values of linear forms on quadratic surfaces

OLIVER SARGENT

Posets, tensor products and Schur positivity

Vyjayanthi Chari, Ghislain Fourier and Daisuke SAGaKi

Parameterizing tropical curves I: Curves of genus zero and one

DAVID E. SPEYER

Pair correlation of angles between reciprocal geodesics on the modular surface

Florin P. Boca, Vicențiu Pașol, AleXandru A. Popa and AleXandru

ZAHARESCU

Étale contractible varieties in positive characteristic

ARMin HolschbaCh, JohanNes SCHMIDT and JaKob STIX 